\title{
Generation of Microsecond Charge-Separated Excited States in Rhenium(I) Diimine Complexes: Driving Force Is the Dominant Factor in Controlling Lifetime
}

Jonathan E. Barnsley, ${ }^{\dagger}$ Georgina E. Shillito, ${ }^{\dagger}$ Christopher B. Larsen, ${ }^{\dagger}$ Holly van der Salm, ${ }^{\dagger}$ Raphael Horvath, ${ }^{\ddagger}$ Xue Zhong Sun, ${ }^{\ddagger}$ Xue Wu, ${ }^{\ddagger}$ Michael W. George, ${ }^{*,+, *}$ Nigel T Lucas ${ }^{*,+}$ and Keith C Gordon*,+

${ }^{+}$Department of Chemistry, University of Otago, P.O. Box 56, Dunedin, New Zealand. Email: keith.gordon@otago.ac.nz,nlucas@chemistry.otago.ac.nz

${ }^{*}$ School of Chemistry, University of Nottingham, Nottingham NG7 2NR, United Kingdom ${ }^{\%}$ Department of Chemical and Environmental Engineering, University of Nottingham Ningbo China, 199 Taikang East Road, Ningbo 315100, China

\footnotetext{
*Correspondence should be addressed to Keith Gordon (keith.gordon@otago.ac.nz), Nigel T. Lucas (nigel.lucas@otago.ac.nz), fax: +64 3479 7906, phone: +64 3 479 5377; and Michael George (mike.george@nottingham.ac.uk), Fax: +115 846 8459. Phone: +115 9513512.
} 

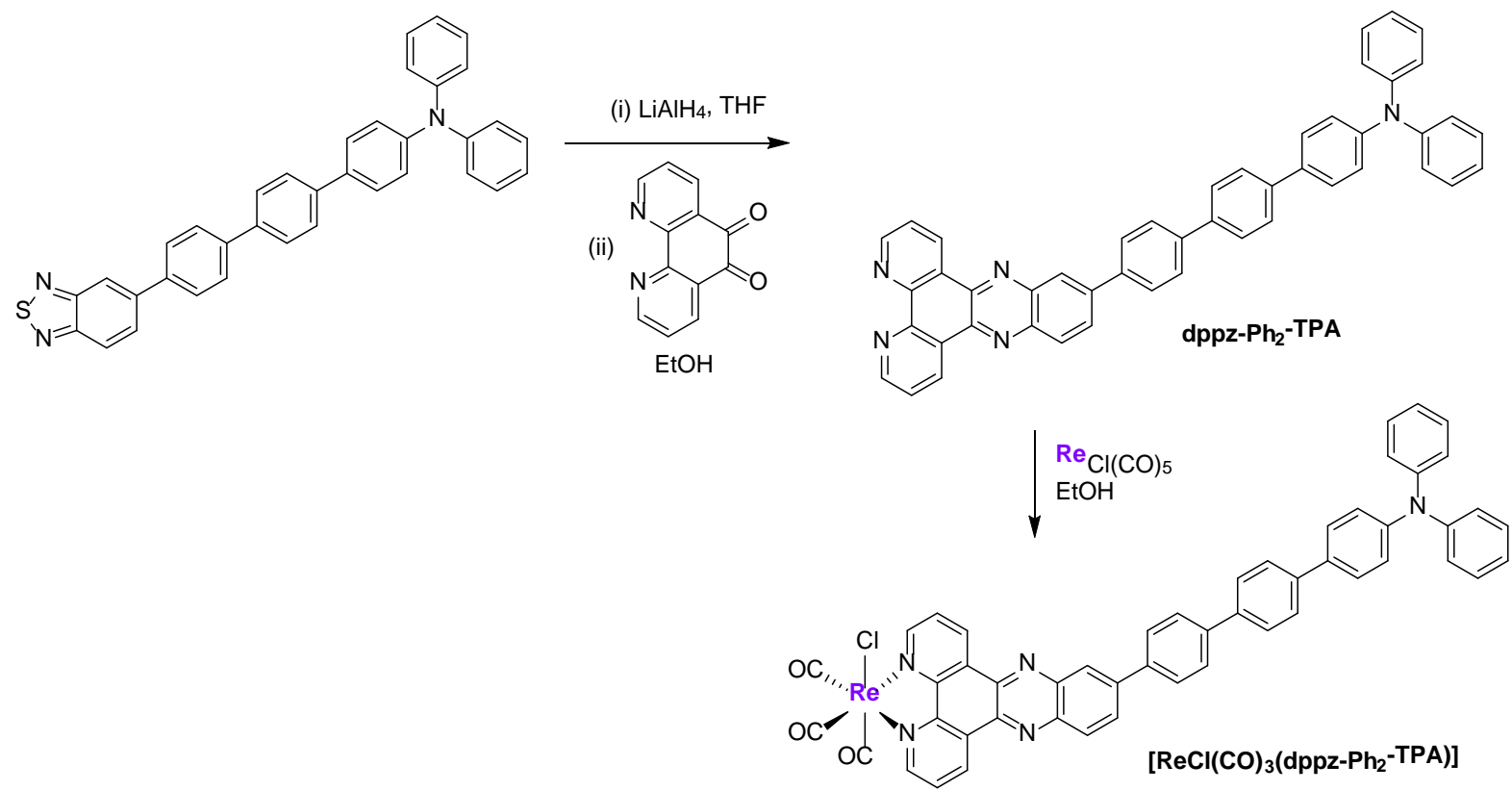

Scheme S1. Synthesis of the dppz-Ph $\mathbf{h}_{2}$-TPA ligand and its reaction with $\operatorname{ReCl}(\mathrm{CO})_{5}$ as a representative example of complexation affording the $\left[\mathrm{ReCl}(\mathrm{CO})_{3}(\mathrm{dppz}-\mathrm{linker-TPA})\right]$ compounds. 
Table S1. Frontier molecular orbitals involved in the lowest energy transitions; these have been drawn with an isovalue of 0.02. dppz-TPA and dppz-NPh $\mathbf{h}_{2}$ orbitals were taken from literature. ${ }^{\mathrm{S} 122}$

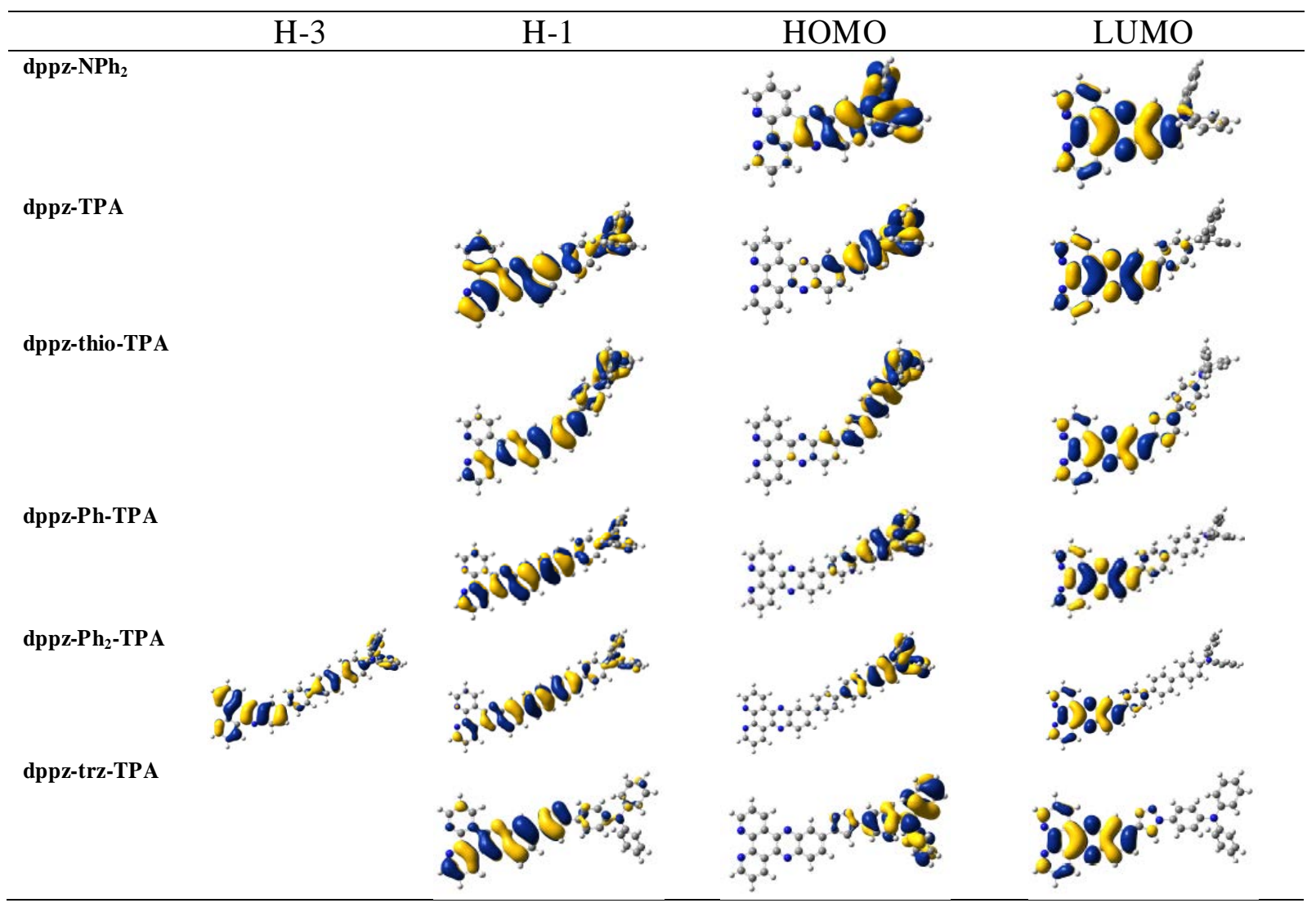

Table S2. Frontier molecular orbitals involved in the lowest energy transitions; these have been drawn with an isovalue of 0.02. $\left[\operatorname{ReCl}(\mathbf{C O})_{3}(\mathbf{d p p z}-\mathrm{TPA})\right]$ and $\left[\operatorname{ReCl}(\mathbf{C O})_{3}\left(\mathbf{d p p z}-\mathrm{NPh}_{2}\right)\right]$ orbitals were taken from literature. ${ }^{\mathrm{S} 1}$

\begin{tabular}{|c|c|c|c|c|c|}
\hline \multirow{2}{*}{$\begin{array}{l}{\left[\mathrm{ReCl}(\mathrm{CO})_{3}(\mathrm{dppz}-\right.} \\
\left.\left.\mathrm{NPh}_{2}\right)\right]\end{array}$} & H-3 & H-2 & H-1 & HOMO & LUMO \\
\hline & & & & & \\
\hline $\begin{array}{l}{\left[\mathrm{ReCl}(\mathrm{CO})_{3}(\mathrm{dppz}-\right.} \\
\mathrm{TPA})]\end{array}$ & & & & & \\
\hline $\begin{array}{l}{\left[\mathrm{ReCl}(\mathrm{CO})_{3}(\mathrm{dppz}-\mathrm{CC}-\right.} \\
\text { TPAA)] }\end{array}$ & & & & & \\
\hline $\begin{array}{l}{\left[\mathrm{ReCl}(\mathrm{CO})_{3}\left(\mathrm{dppz}^{-}\right.\right.} \\
\text {thio-TPA)] }\end{array}$ & & & & & \\
\hline $\begin{array}{l}{\left[\mathrm{ReCl}(\mathrm{CO})_{3}(\mathrm{dppz}-\mathrm{Ph}-\right.} \\
\text { TPA)] }\end{array}$ & & & & & \\
\hline $\begin{array}{l}{\left[\mathrm{ReCl}(\mathrm{CO})_{3}\left(\mathrm{dppz}_{\mathrm{Ph}} \mathrm{Ph}_{2}\right.\right.} \\
\text { TPA })]\end{array}$ & & & & & \\
\hline $\begin{array}{l}{\left[\mathrm{ReCl}(\mathrm{CO})_{3}(\mathrm{dppz}-\mathrm{trz}-\right.} \\
\text { TPA)] }\end{array}$ & $\therefore$ & & & & \\
\hline
\end{tabular}


Table S3. Neat FT-Raman data with assignments.

\begin{tabular}{|c|c|c|c|c|c|}
\hline Ligand & $\begin{array}{c}\text { Raman Shift } \\
/ \mathrm{cm}^{-1}\end{array}$ & Assignment & Complex & $\begin{array}{l}\text { Raman Shi } \\
/ \mathrm{cm}^{-1}\end{array}$ & Assignment \\
\hline \multirow[t]{13}{*}{ dppz-NPh } & 1621 & $\mathrm{NPh}_{2}-\mathrm{phz}$ & {$\left[\operatorname{ReCl}(\mathrm{CO})_{3}\left(\mathrm{dppz}-\mathrm{NPh}_{2}\right)\right]$} & 1921 & carbonyl \\
\hline & 1590 & mixed & & 1896 & carbonyl \\
\hline & 1527 & phz & & 1604 & $\mathrm{NPh}_{2}-\mathrm{phz}$ \\
\hline & 1471 & dppz & & 1575 & phz \\
\hline & 1459 & mixed & & 1528 & phz \\
\hline & 1414 & phen & & 1496 & dppz \\
\hline & 1354 & dppz & & 1447 & $\mathrm{NPh}_{2}$-dppz \\
\hline & 1322 & dppz & & 1419 & phen \\
\hline & 1234 & phz & & 1356 & dppz \\
\hline & 1031 & phen & & 1323 & dppz \\
\hline & 1000 & $\mathrm{NPh}_{2}$ & & 1236 & phz \\
\hline & & & & 1054 & dppz \\
\hline & & & & 1002 & $\mathrm{NPh}_{2}$ \\
\hline \multirow[t]{12}{*}{ dppz-TPA } & 1608 & TPA & {$\left[\operatorname{ReCl}(\mathrm{CO})_{3}(\mathrm{dppz}-\mathrm{TPA})\right]$} & 1913 & carbonyl \\
\hline & 1594 & TPA & & 1592 & TPA \\
\hline & 1531 & phz & & 1521 & phz \\
\hline & 1468 & dppz & & 1449 & mixed \\
\hline & 1446 & mixed & & 1416 & phen \\
\hline & 1409 & phen & & 1324 & dppz \\
\hline & 1324 & dppz & & 1253 & dppz \\
\hline & 1264 & mixed & & 1208 & TPA \\
\hline & 1205 & TPA & & 1054 & dppz \\
\hline & 1177 & mixed & & 1001 & TPA \\
\hline & 1031 & phen & & & \\
\hline & 999 & TPA & & & \\
\hline \multirow[t]{13}{*}{ dppz-CC-TPA } & 2202 & alkyne & {$\left[\operatorname{ReCl}(\mathrm{CO})_{3}(\mathrm{dppz}-\mathrm{CC}-\mathrm{TPA})\right]$} & 2201 & alkyne \\
\hline & 1605 & TPA & & 1922 & carbonyl \\
\hline & 1589 & mixed & & 1893 & carbonyl \\
\hline & 1534 & phz & & 1601 & TPA \\
\hline & 1468 & dppz & & 1589 & mixed \\
\hline & 1441 & mixed & & 1536 & phz \\
\hline & 1407 & phen & & 1447 & mixed \\
\hline & 1346 & dppz & & 1411 & phen \\
\hline & 1114 & linker & & 1349 & dppz \\
\hline & 1031 & phen & & 1320 & dppz \\
\hline & 1000 & TPA & & 1113 & linker \\
\hline & & & & 1053 & dppz \\
\hline & & & & 1001 & TPA \\
\hline \multirow[t]{10}{*}{ dppz-thio-TPA } & 1612 & TPA & {$\left[\operatorname{ReCl}(\mathrm{CO})_{3}(\right.$ dppz-thio-TPA $\left.)\right]$} & 1907 & carbonyl \\
\hline & 1595 & TPA & & 1597 & TPA \\
\hline & 1510 & mixed & & 1508 & mixed \\
\hline & 1473 & dppz & & 1438 & thiophene \\
\hline & 1443 & thiophene & & 1419 & phen \\
\hline & 1342 & dppz & & 1316 & dppz \\
\hline & 1195 & TPA & & 1195 & TPA \\
\hline & 1180 & mixed & & 1176 & mixed \\
\hline & 1063 & thiophene & & 1077 & thiophene \\
\hline & 1001 & TPA & & 1000 & TPA \\
\hline \multirow[t]{10}{*}{ dppz-Ph-TPA } & 1605 & TPA & {$\left[\operatorname{ReCl}(\mathrm{CO})_{3}(\mathrm{dppz}-\mathrm{Ph}-\mathrm{TPA})\right]$} & 1923 & carbonyl \\
\hline & 1531 & phz & & 1887 & carbonyl \\
\hline & 1447 & mixed & & 1601 & TPA \\
\hline & 1410 & phen & & 1533 & phz \\
\hline & 1287 & linker & & 1448 & mixed \\
\hline & 1264 & mixed & & 1410 & phen \\
\hline & 1244 & dppz & & 1287 & linker \\
\hline & 1203 & TPA & & 1205 & TPA \\
\hline & 1031 & phen & & 1053 & dppz \\
\hline & 1001 & TPA & & 1000 & TPA \\
\hline \multirow[t]{8}{*}{ dppz-Ph ${ }_{2}$-TPA } & 1602 & TPA & {$\left[\operatorname{ReCl}(\mathrm{CO})_{3}\left(\mathrm{dppz}-\mathrm{Ph}_{2}-\mathrm{TPA}\right)\right]$} & 1600 & TPA \\
\hline & 1533 & phz & & 1531 & phz \\
\hline & 1468 & dppz & & 1447 & mixed \\
\hline & 1447 & mixed & & 1412 & phen \\
\hline & 1411 & phen & & 1283 & linker \\
\hline & 1284 & linker & & 1245 & dppz \\
\hline & 1245 & dppz & & 1206 & TPA \\
\hline & 1206 & TPA & & 1053 & dppz \\
\hline
\end{tabular}




\begin{tabular}{|c|c|c|c|c|c|}
\hline & 1002 & TPA & & 1001 & TPA \\
\hline \multirow[t]{13}{*}{ dppz-trz-TPA } & 1617 & TPA & {$\left[\operatorname{ReCl}(\mathrm{CO})_{3}(\right.$ dppz-trz-TPA)] } & 1928 & carbonyl \\
\hline & 1590 & mixed & & 1897 & carbonyl \\
\hline & 1541 & phz & & 1613 & TPA \\
\hline & 1504 & mixed & & 1540 & phz \\
\hline & 1473 & mixed & & 1499 & mixed \\
\hline & 1419 & phen & & 1449 & mixed \\
\hline & 1339 & dppz & & 1425 & phen \\
\hline & 1320 & dppz & & 1342 & dppz \\
\hline & 1182 & mixed & & 1313 & dppz \\
\hline & 1031 & triazole & & 1185 & mixed \\
\hline & 1001 & TPA & & 1054 & triazole \\
\hline & 965 & triazole & & 1001 & TPA \\
\hline & & & & 963 & triazole \\
\hline
\end{tabular}

Table S4 Electrochemical data for investigated compounds $\left(\mathrm{CH}_{2} \mathrm{Cl}_{2}, 0.1 \mathrm{M} \mathrm{NBu}_{4} \mathrm{PF}_{6}\right.$ electrolyte), referenced against SCE using the $\left(\mathrm{FC}^{*}\right)^{+} / \mathrm{FC}^{*}$ couple as an internal standard. Bandgap generated using a method by Li et al. ${ }^{\mathrm{S} 1}$

\begin{tabular}{|c|c|c|c|c|}
\hline \multirow[b]{2}{*}{ Compound } & \multicolumn{4}{|c|}{$\mathrm{E}^{\circ} / \mathrm{V}$ vs $\operatorname{SCE}(\Delta R)$} \\
\hline & $E_{\text {red }}$ & $\mathrm{E}_{\mathrm{OX}}$ & $\mathrm{E}_{\text {ox-red }}$ & Bandgap / eV \\
\hline dppz-NPh 2 & -1.29 & 1.19 & 2.48 & 2.58 \\
\hline$\left[\operatorname{ReCl}(\mathrm{CO})_{3}\left(\mathrm{dppz}^{\left.\left.-\mathrm{NPh}_{2}\right)\right]}\right.\right.$ & $-1.06(0.23)$ & $1.19(0.00)$ & $2.25(0.23)$ & 2.42 \\
\hline dppz-TPA & -1.23 & 1.03 & 2.26 & 2.38 \\
\hline$\left[\operatorname{ReCl}(\mathrm{CO})_{3}(\operatorname{dppz}-\mathrm{TPA})\right]$ & $-0.97(0.26)$ & $1.05(0.02)$ & $2.02(0.24)$ & 2.13 \\
\hline dppz-CC-TPA & -1.16 & 1.04 & 2.20 & 2.33 \\
\hline$\left[\operatorname{ReCl}(\mathrm{CO})_{3}(\mathrm{dppz}-\mathrm{CC}-\mathrm{TPA})\right]$ & $-0.91(0.25)$ & $1.06(0.02)$ & $1.97(0.23)$ & 2.06 \\
\hline dppz-thio-TPA & -1.23 & 0.90 & 2.13 & 2.23 \\
\hline$\left[\operatorname{ReCl}(\mathrm{CO})_{3}(\right.$ dppz-thio-TPA)] & $-0.95(0.28)$ & $0.94(0.04)$ & $1.89(0.24)$ & 1.99 \\
\hline dppz-Ph-TPA & -1.21 & 0.99 & 2.20 & 2.29 \\
\hline$\left[\operatorname{ReCl}(\mathrm{CO})_{3}(\mathrm{dppz}-\mathrm{Ph}-\mathrm{TPA})\right]$ & $-0.97(0.24)$ & $1.00(0.01)$ & $1.97(0.23)$ & 2.11 \\
\hline dppz-Ph - TPA & -1.26 & 0.97 & 2.23 & 2.30 \\
\hline$\left[\operatorname{ReCl}(\mathrm{CO})_{3}\left(\mathrm{dppz}-\mathrm{Ph}_{2}-\mathrm{TPA}\right)\right]$ & $-0.97(0.29)$ & $0.97(0.00)$ & $1.94(0.29)$ & 2.05 \\
\hline dppz-trz-TPA & -1.10 & 1.01 & 2.11 & 2.24 \\
\hline$\left[\operatorname{ReCl}(\mathrm{CO})_{3}(\mathrm{dppz}-\operatorname{trz}-\mathrm{TPA})\right]$ & $-0.86(0.24)$ & $1.00(0.01)$ & $1.86(0.25)$ & 1.98 \\
\hline
\end{tabular}

Table S5 Mean absolute deviation and associated scaling factor for Raman data.

\begin{tabular}{|c|c|c|c|c|}
\hline \multirow[b]{2}{*}{ Compound } & \multicolumn{2}{|c|}{ B3LYP } & \multicolumn{2}{|c|}{ CAM-B3LYP } \\
\hline & $\mathrm{MAD} / \mathrm{cm}^{-1}$ & Scaling Factor & $\mathrm{MAD} / \mathrm{cm}^{-1}$ & Scaling Factor \\
\hline dppz-NPh 2 & 3.36 & 0.975 & 4.04 & 0.950 \\
\hline$\left[\operatorname{ReCl}(\mathrm{CO})_{3}\left(\mathrm{dppz}-\mathrm{NPh}_{2}\right)\right]$ & 5.78 & 0.965 & 4.14 & 0.948 \\
\hline dppz-TPA & 2.71 & 0.971 & 4.78 & 0.951 \\
\hline$\left[\operatorname{ReCl}(\mathrm{CO})_{3}(\mathrm{dppz}-\mathrm{TPA})\right]$ & 4.48 & 0.967 & 8.22 & 0.944 \\
\hline dppz-CC-TPA & 3.48 & 0.968 & 5.10 & 0.945 \\
\hline$\left[\operatorname{ReCl}(\mathrm{CO})_{3}(\mathrm{dppz}-\mathrm{CC}-\mathrm{TPA})\right]$ & 3.35 & 0.969 & 5.28 & 0.945 \\
\hline dppz-thio-TPA & 2.65 & 0.972 & 6.37 & 0.956 \\
\hline$\left[\operatorname{ReCl}(\mathrm{CO})_{3}(\right.$ dppz-thio-TPA)] & 4.59 & 0.967 & 5.03 & 0.950 \\
\hline dppz-Ph-TPA & 5.06 & 0.969 & 5.73 & 0.953 \\
\hline$\left[\operatorname{ReCl}(\mathrm{CO})_{3}(\mathrm{dppz}-\mathrm{Ph}-\mathrm{TPA})\right]$ & 7.40 & 0.960 & 5.94 & 0.945 \\
\hline dppz-Ph ${ }_{2}$-TPA & 3.97 & 0.977 & 6.08 & 0.952 \\
\hline$\left[\operatorname{ReCl}(\mathrm{CO})_{3}\left(\mathrm{dppz}-\mathrm{Ph}_{2}-\mathrm{TPA}\right)\right]$ & 4.44 & 0.972 & 6.54 & 0.951 \\
\hline dppz-trz-TPA & 3.09 & 0.907 & 3.34 & 0.951 \\
\hline$\left[\operatorname{ReCl}(\mathrm{CO})_{3}(\right.$ dppz-trz-TPA)] & 5.70 & 0.965 & 4.09 & 0.951 \\
\hline
\end{tabular}


Table S1 Lifetime data for the longest lived state, measured using transient absorption and TR-IR. Data collected in $\mathrm{CH}_{2} \mathrm{Cl}_{2}$ at room temperature.

\begin{tabular}{|c|c|c|c|c|}
\hline Compound & $\begin{array}{l}\text { Transient Absorption } \\
\text { Lifetime / ns }\end{array}$ & $\begin{array}{c}\text { TR-IR } \\
\text { Lifetime / ns }\end{array}$ & $\begin{array}{l}\text { CO Frequency } \\
/ \mathrm{cm}^{-1}\end{array}$ & Ligand Frequency $/ \mathrm{cm}^{-1}$ \\
\hline$\left[\operatorname{ReCl}(\mathrm{CO})_{3}\left(\mathrm{dppz}-\mathrm{NPh}_{2}\right)\right]$ & $6,100 \pm 1000$ & $2300 \pm 500$ & 2020,1891 & $1563,1537,1507,1404$ \\
\hline$\left[\operatorname{ReCl}(\mathrm{CO})_{3}(\mathrm{dppz}-\mathrm{TPA})\right]$ & $3,800 \pm 400$ & $2200 \pm 600$ & 2019, 1887 & 1537, 1504, $1489,1479,1341$ \\
\hline$\left[\operatorname{ReCl}(\mathrm{CO})_{3}(\mathrm{dppz}-\mathrm{CC}-\mathrm{TPA})\right]$ & $3,200 \pm 800$ & - & - & - \\
\hline$\left[\operatorname{ReCl}(\mathrm{CO})_{3}(\right.$ dppz-thio-TPA)] & $800 \pm 100$ & $1280 \pm 50$ & 2019, 1887 & 1551 \\
\hline$\left[\operatorname{ReCl}(\mathrm{CO})_{3}(\mathrm{dppz}-\mathrm{Ph}-\mathrm{TPA})\right]$ & $2,100 \pm 700$ & $1280 \pm 30$ & 2019, 1887 & 1535, 1476 \\
\hline$\left[\operatorname{ReCl}(\mathrm{CO})_{3}\left(\mathrm{dppz}-\mathrm{Ph}_{2}-\mathrm{TPA}\right)\right]$ & $1,200 \pm 200$ & $950 \pm 90$ & 2019, 1887 & 1506 \\
\hline$\left[\operatorname{ReCl}(\mathrm{CO})_{3}(\mathrm{dppz}-\operatorname{trz}-\mathrm{TPA})\right]$ & $600 \pm 200$ & $800 \pm 300^{*}$ & 2019, 1891 & $\begin{array}{l}\text { 1591, 1567, 1545, 1536, 1335, } \\
1326\end{array}$ \\
\hline
\end{tabular}




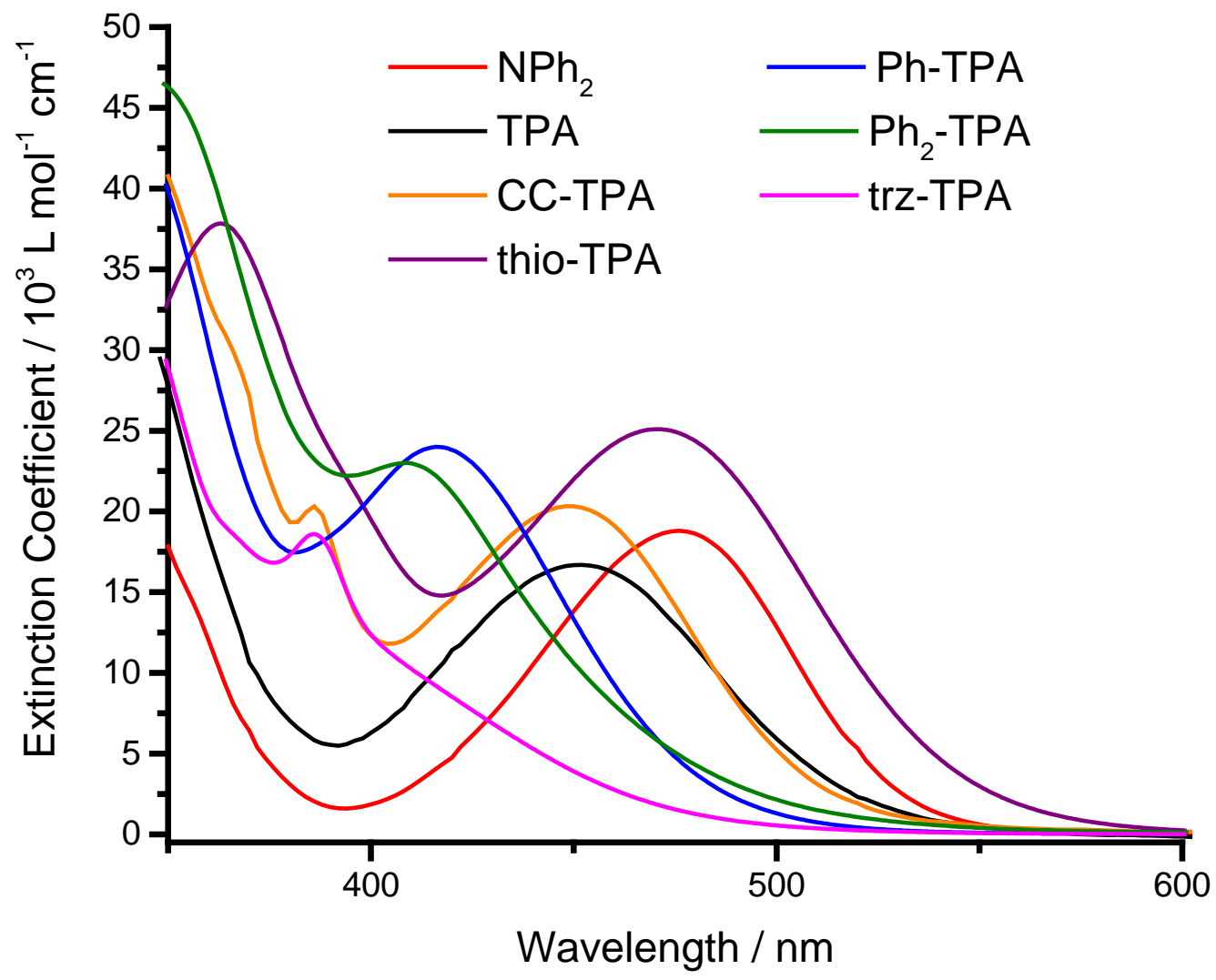

Figure S1. UV-vis spectra for the ligands in $\mathrm{CH}_{2} \mathrm{Cl}_{2}$. dppz-TPA and dppz-NPh2 spectra were taken from literature. ${ }^{\mathrm{S} 2}$ 


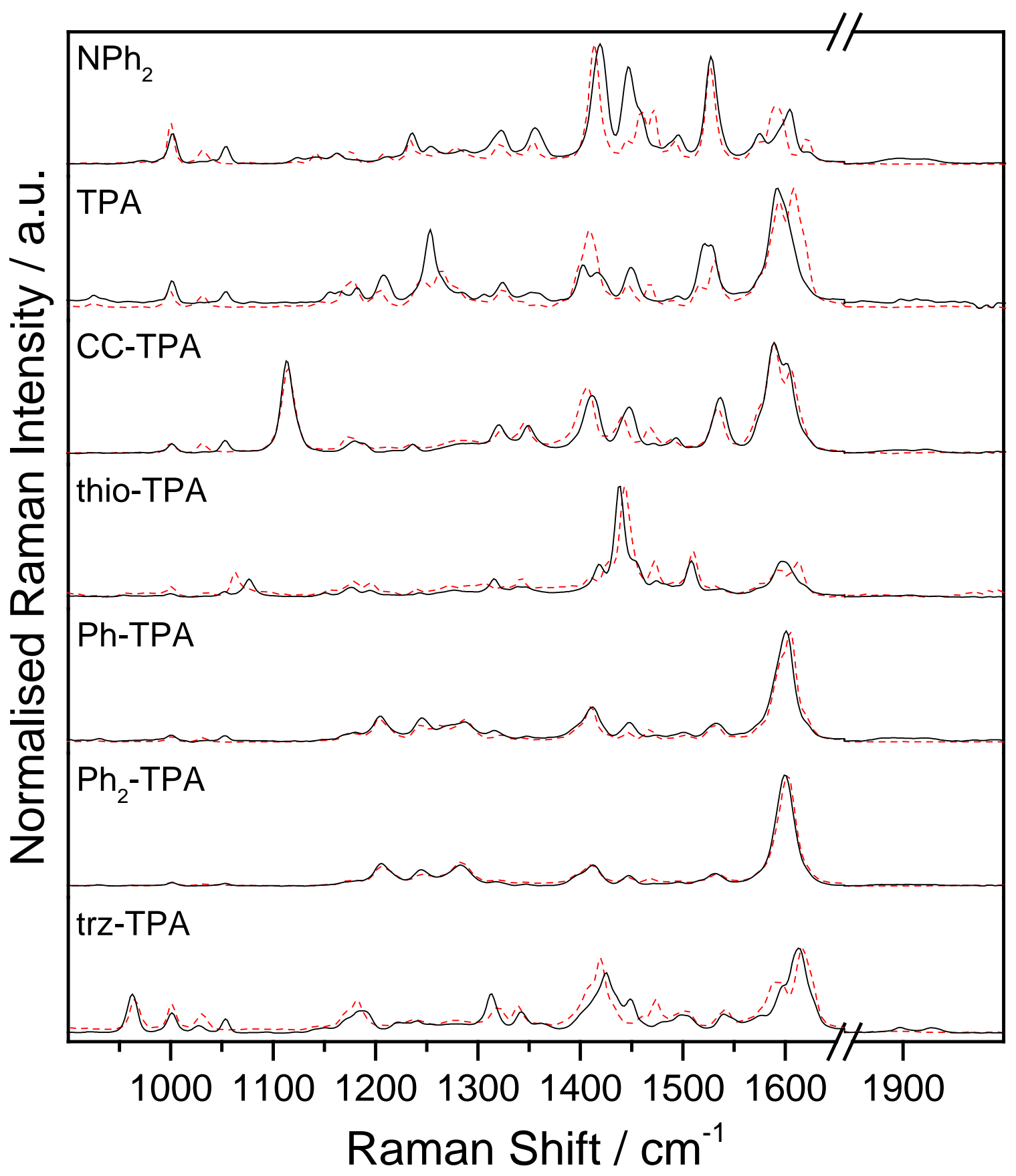

Figure S2. Neat FT-Raman spectra for the ligands (red dashed line) and complexes (black line). dppz-TPA and dppz-NPh ligand and complex spectra were taken from literature. ${ }^{\mathrm{s} 1}$ 
a) dppz-NPh 2

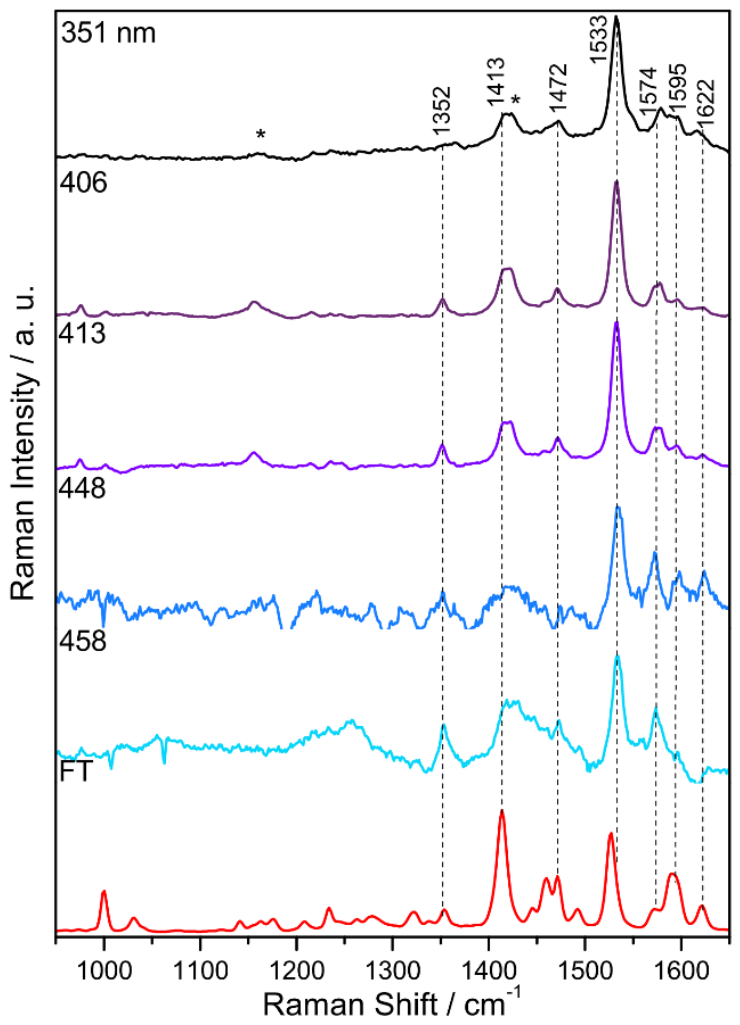

c) dppz-TPA

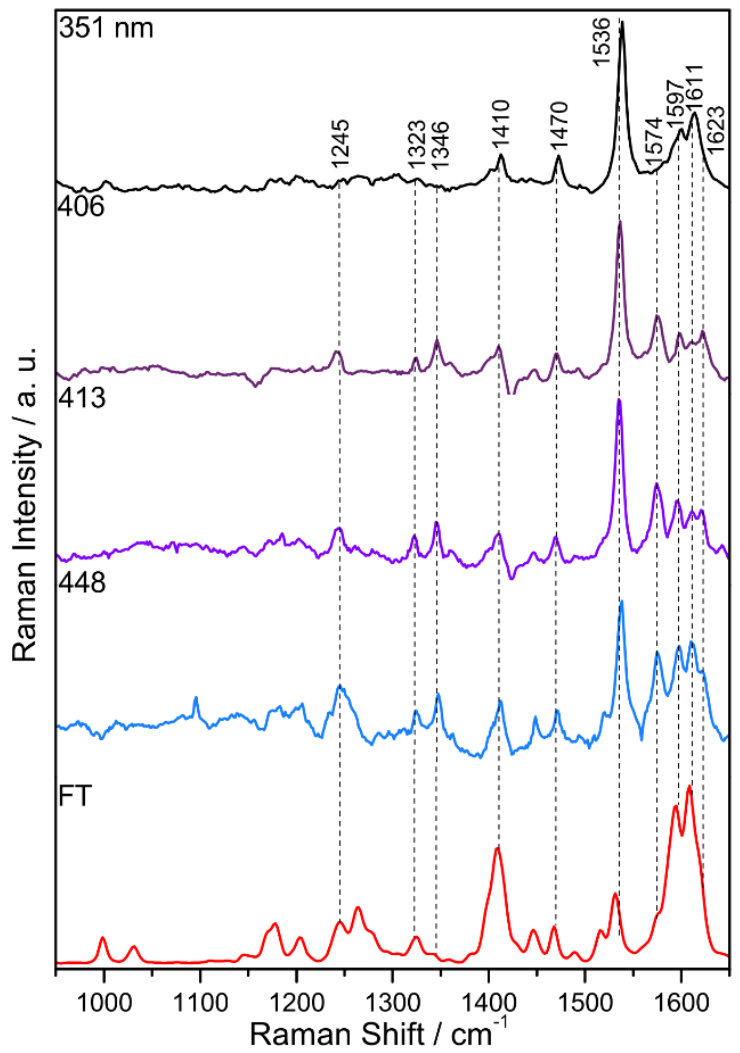

b) $\left[\operatorname{ReCl}(\mathrm{CO})_{3}\left(\mathrm{dppz}-\mathrm{NPh}_{2}\right)\right]$

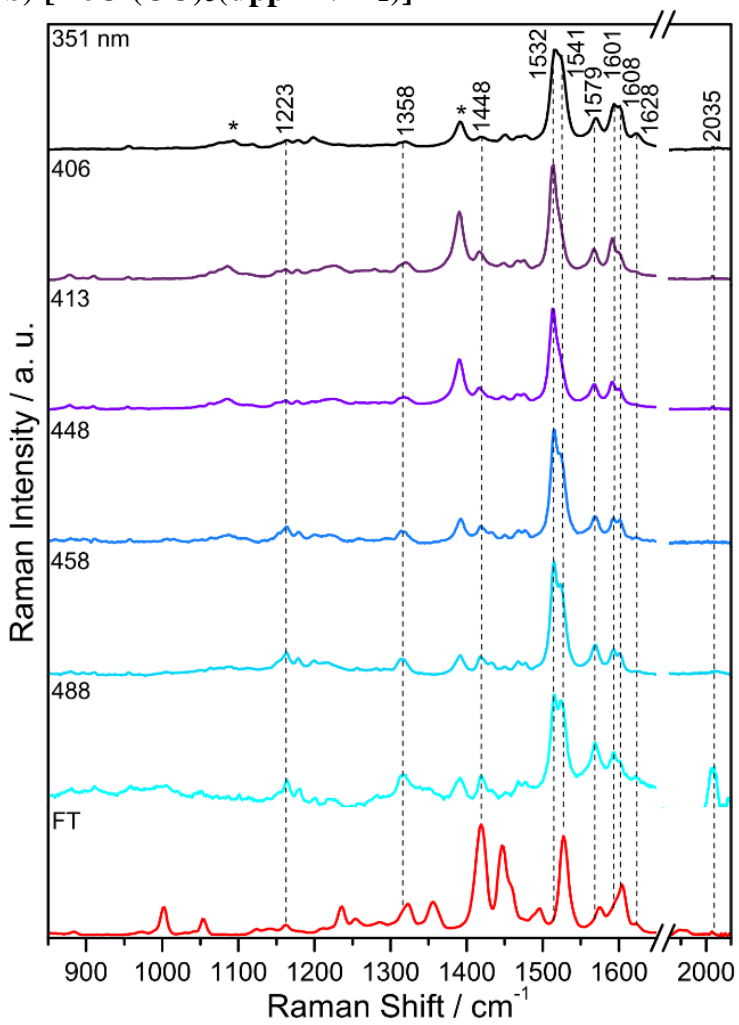

d) $\left[\operatorname{ReCl}(\mathrm{CO})_{3}(\mathrm{dppz}-\mathrm{TPA})\right]$

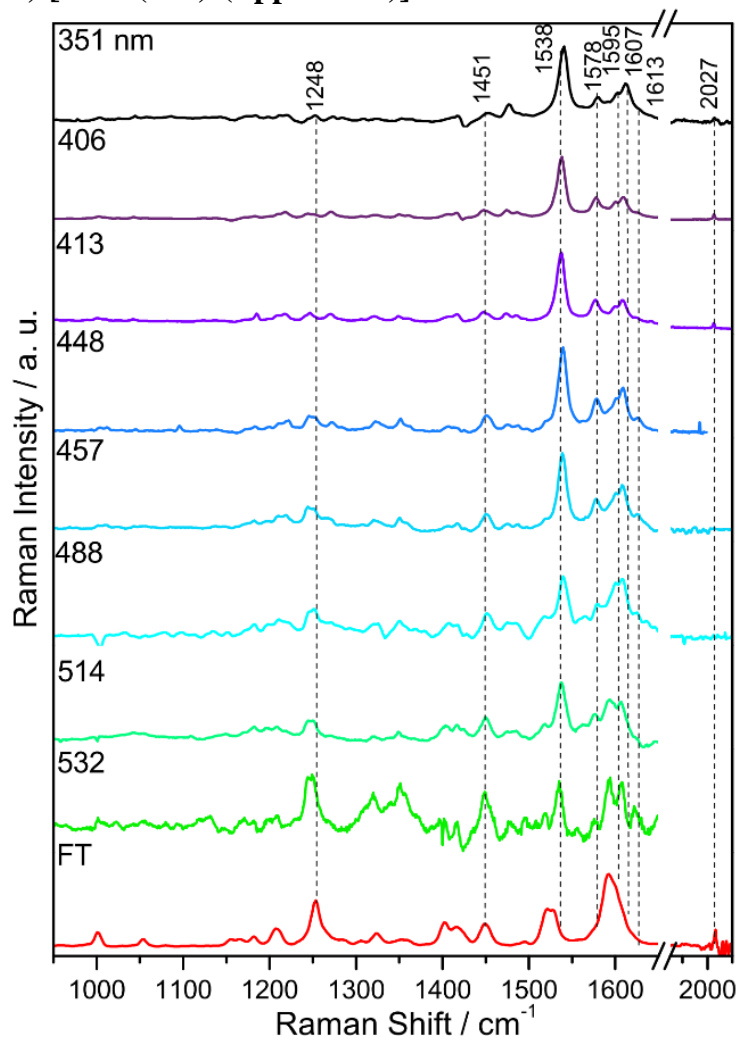

Figure S1 Resonance and non-resonance Raman data collected using a number of excitation wavelengths in $\mathrm{CH}_{2} \mathrm{Cl}_{2}$ at $10^{-3}$ $\mathrm{M}$. Bands of interest are shown with a dropline and * denotes solvent bands. Spectra taken from literature. ${ }^{\mathrm{S} 1}$ 
a) dppz-CC-TPA

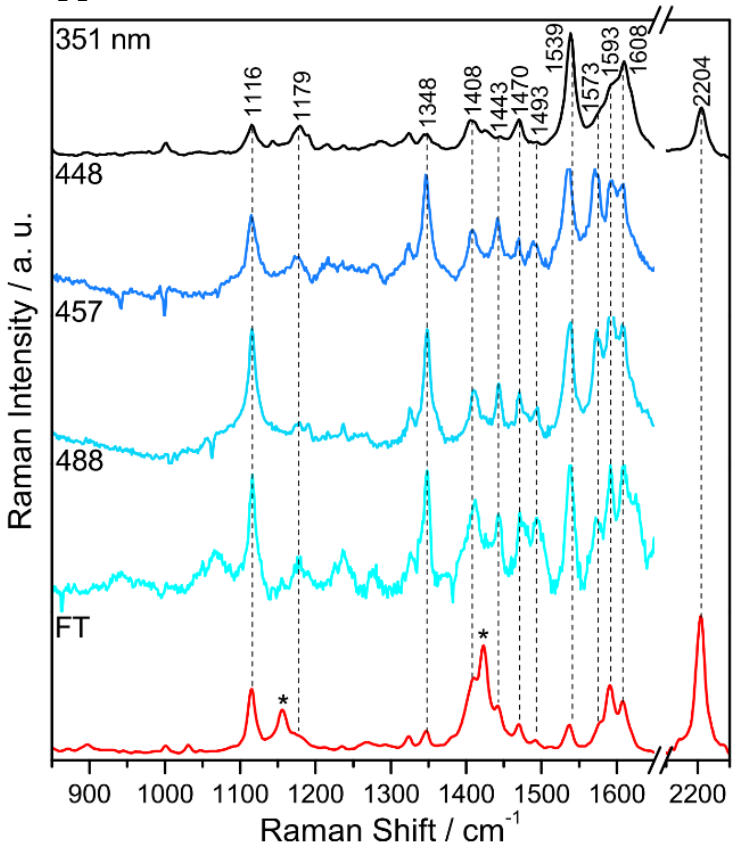

c) dppz-Ph-TPA

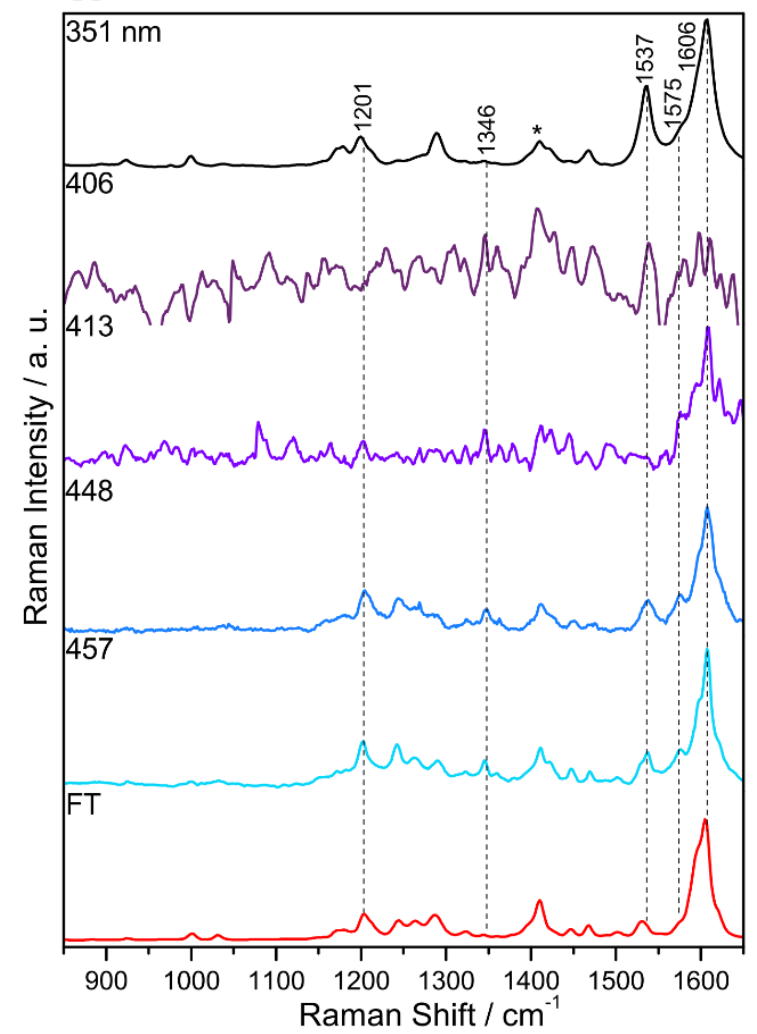

b) $\left[\operatorname{ReCl}(\mathrm{CO})_{3}(\mathrm{dppz}-\mathrm{CC}-\mathrm{TPA})\right]$

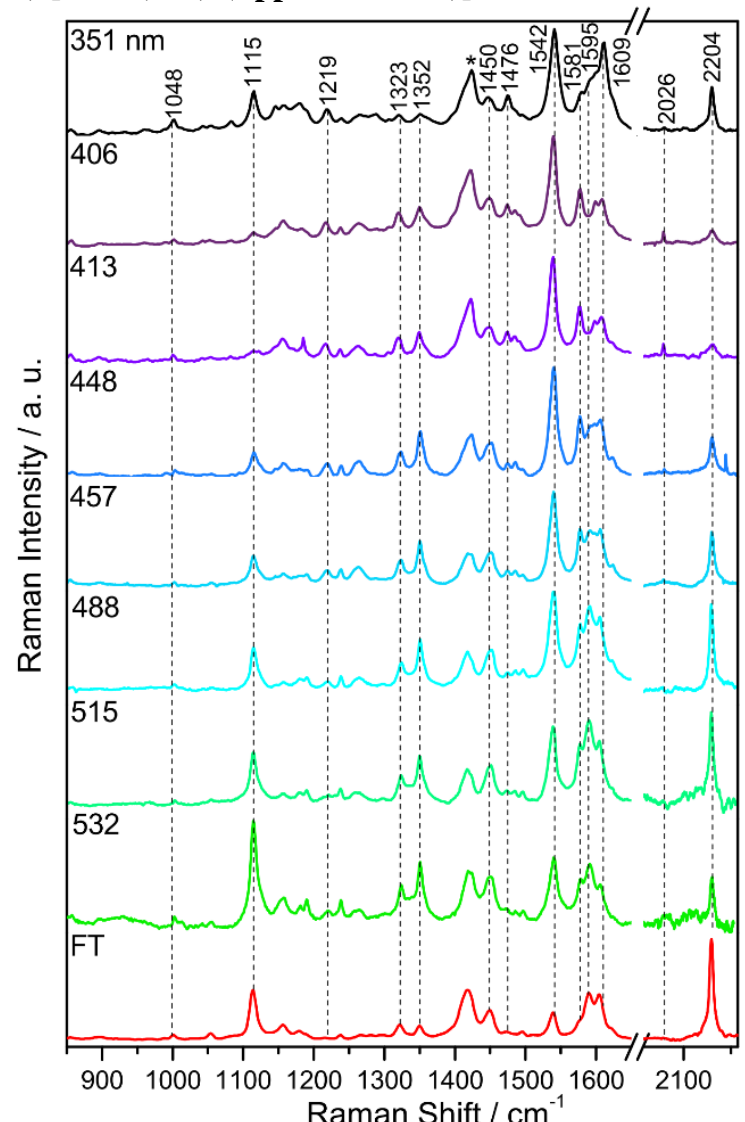

d) $\left[\operatorname{ReCl}(\mathrm{CO})_{3}(\mathrm{dppz}-\mathrm{Ph}-\mathrm{TPA})\right]$

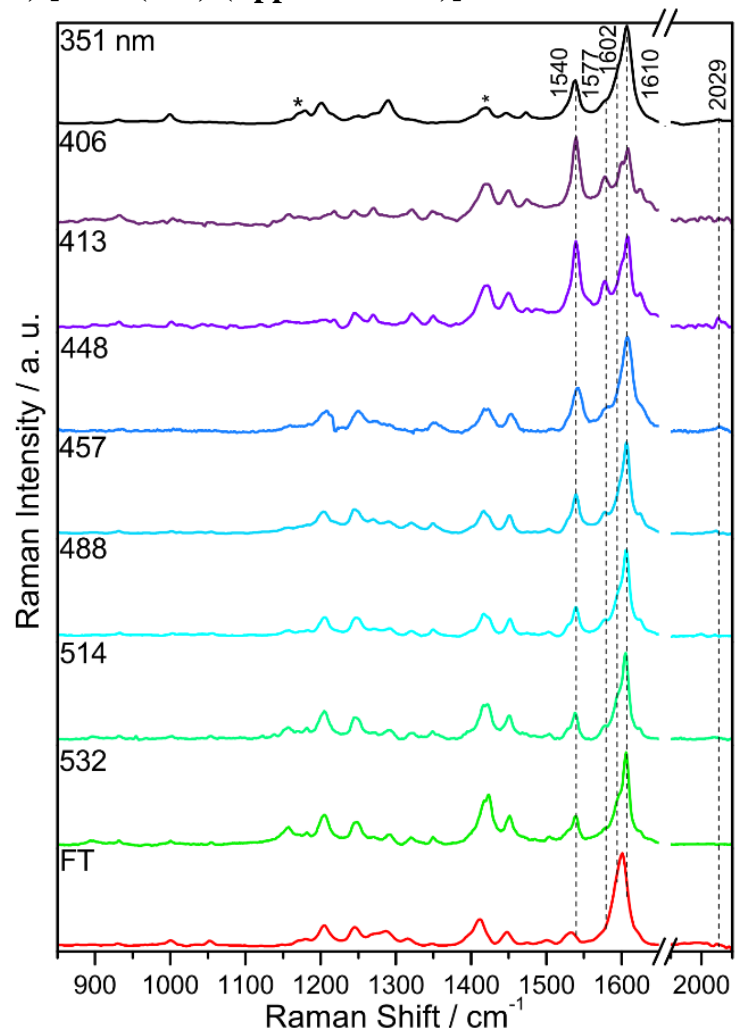

Figure S2 Resonance and non-resonance Raman data collected using a number of excitation wavelengths in $\mathrm{CH}_{2} \mathrm{Cl}_{2}$ at $10^{-3}$ M. Bands of interest are shown with a dropline and * denotes solvent bands 
a) dppz-Ph2-TPA

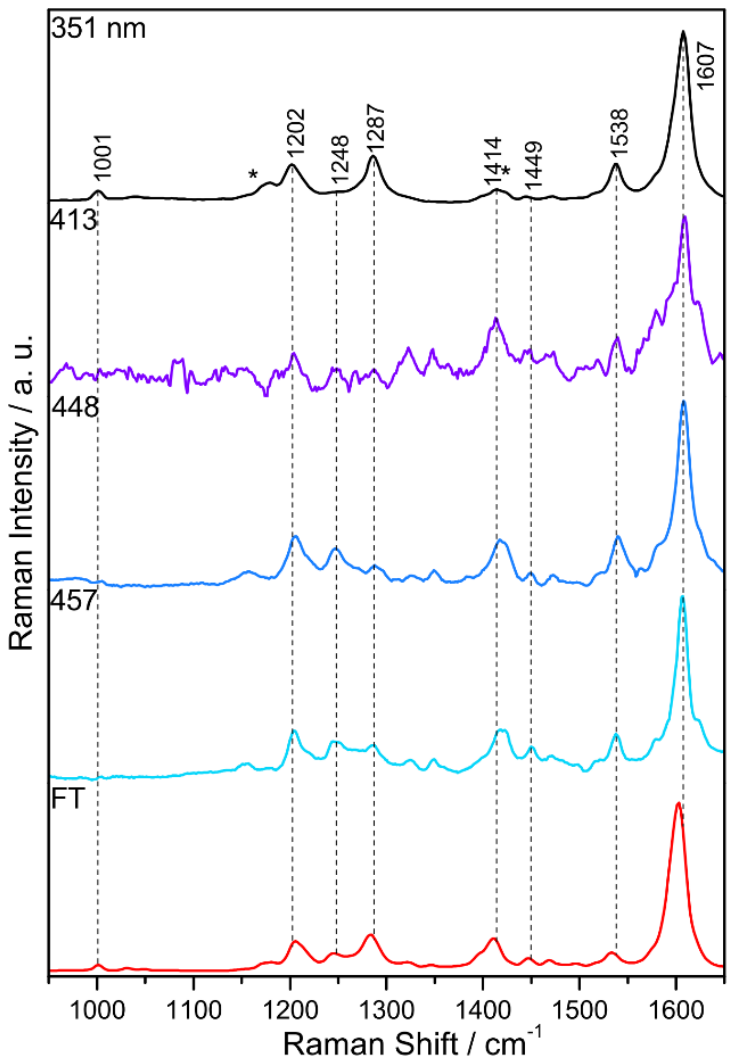

c) dppz-thio-TPA

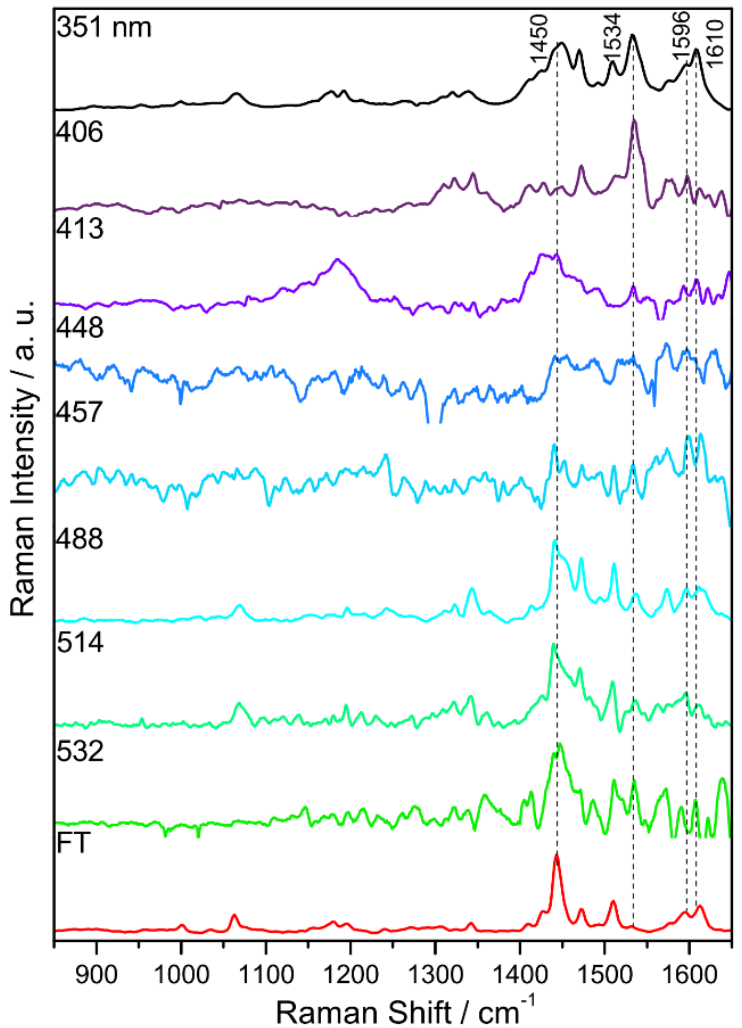

b) $\left[\operatorname{ReCl}(\mathrm{CO})_{3}\left(\mathrm{dppz}-\mathrm{Ph}_{2}-\mathrm{TPA}\right)\right]$

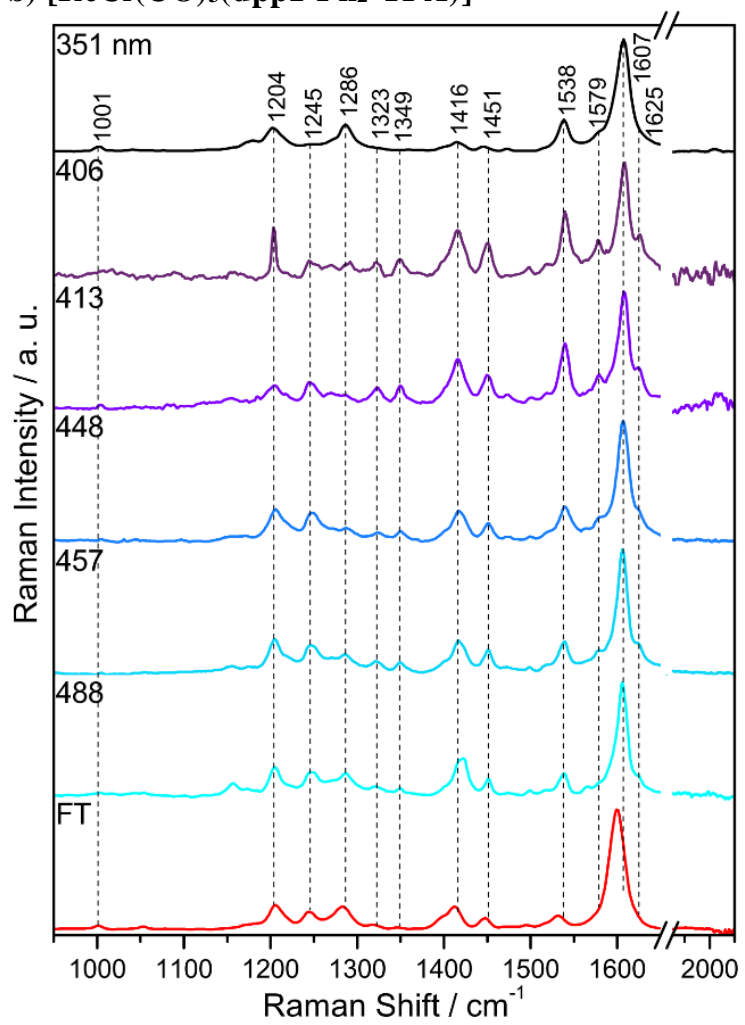

d) $\left[\operatorname{ReCl}(\mathrm{CO})_{3}(\right.$ dppz-thio-TPA $\left.)\right]$

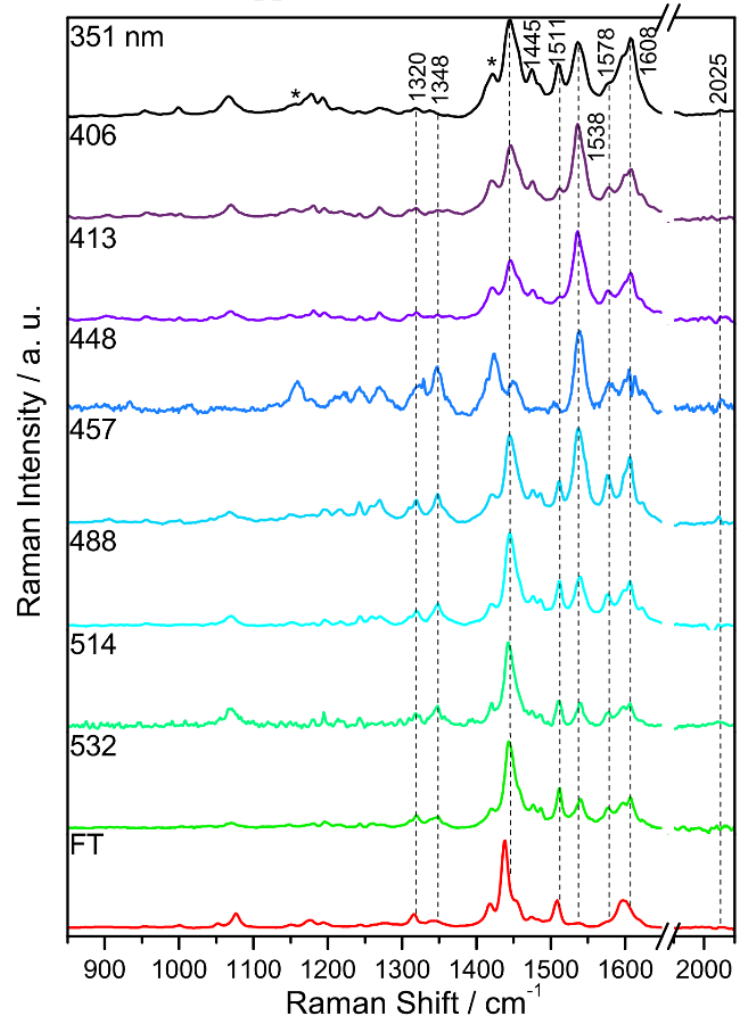

Figure S3 Resonance and non-resonance Raman data collected using a number of excitation wavelengths in $\mathrm{CH}_{2} \mathrm{Cl}_{2}$ at $10^{-3}$ M. Bands of interest are shown with a dropline and * denotes solvent bands 
a) dppz-trz-TPA

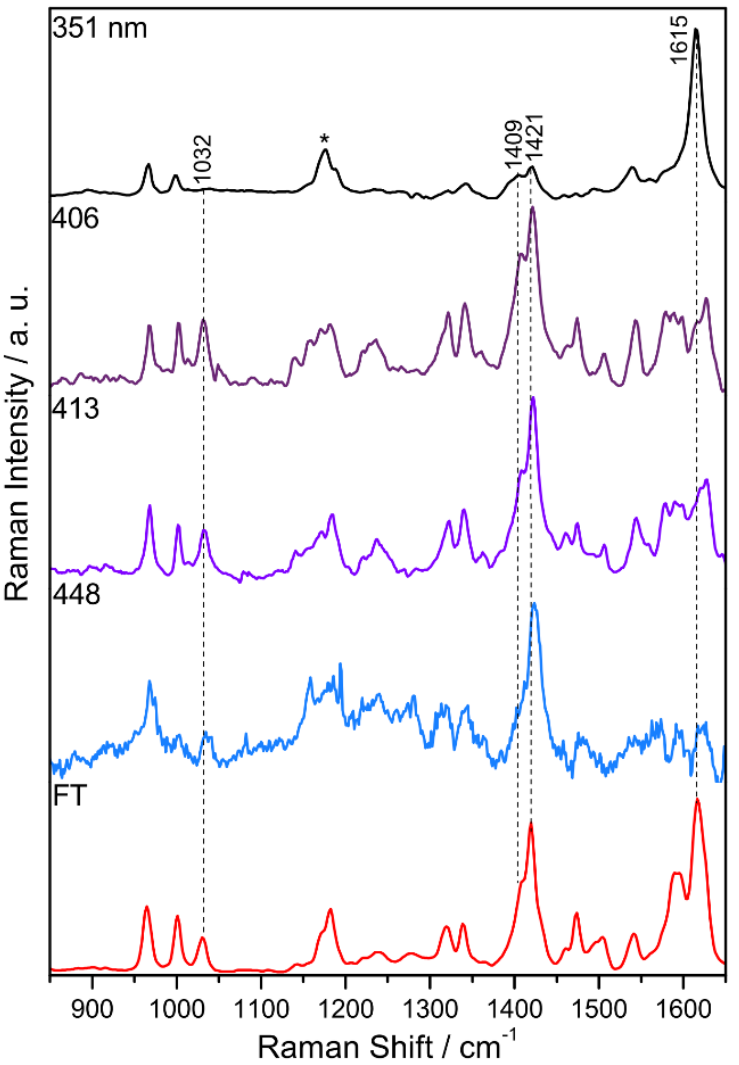

b) $\left[\mathrm{ReCl}(\mathrm{CO})_{3}(\mathrm{dppz}-\operatorname{trz}-\mathrm{TPA})\right]$

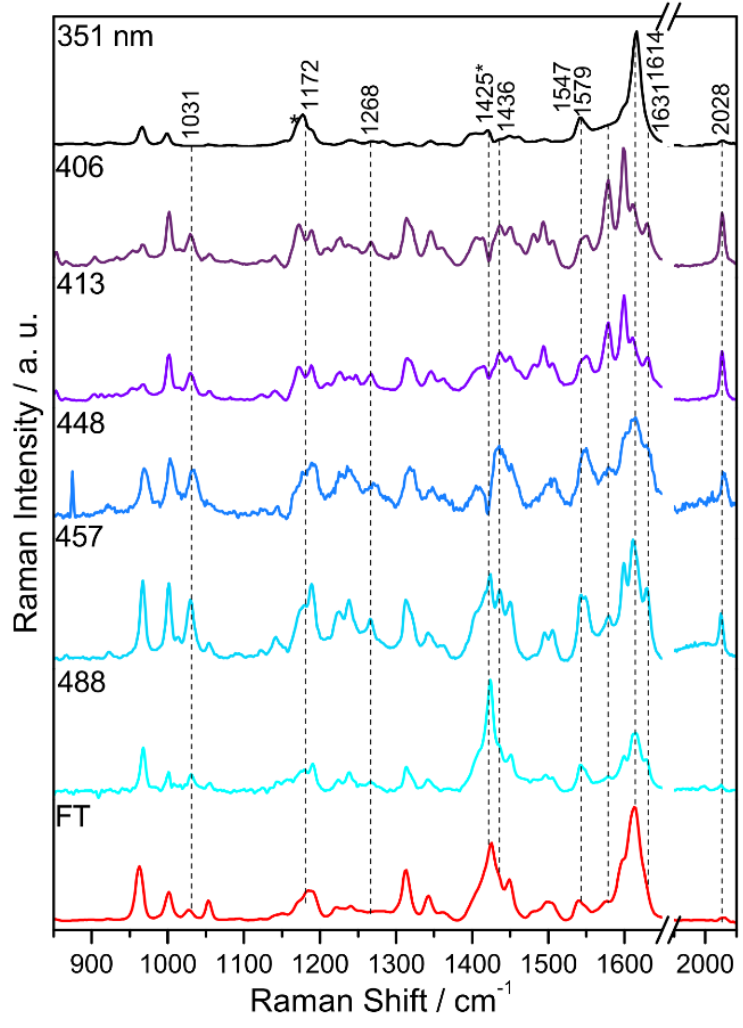

Figure S4 Resonance and non-resonance Raman data collected using a number of excitation wavelengths in $\mathrm{CH}_{2} \mathrm{Cl}_{2}$ at $10^{-3}$ M. Bands of interest are shown with a dropline and * denotes solvent bands

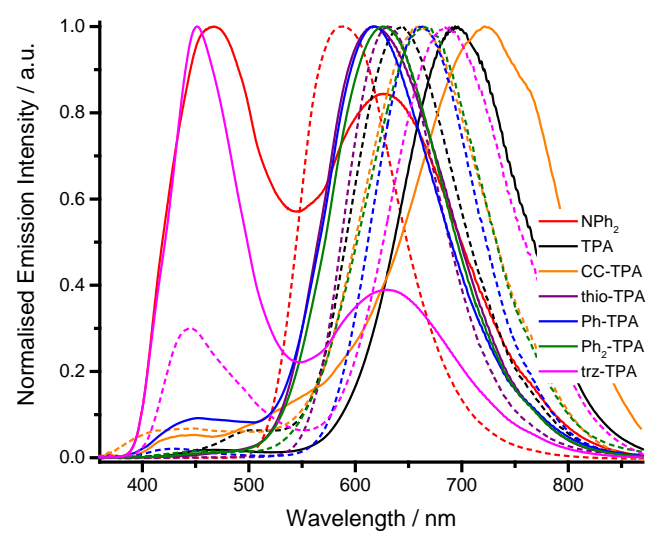

Figure S7 Emission data for the ligands (dashed line) and complexes (solid line) in chlorobenzene at $\sim 5 \times 10^{-5} \mathrm{M}$. 


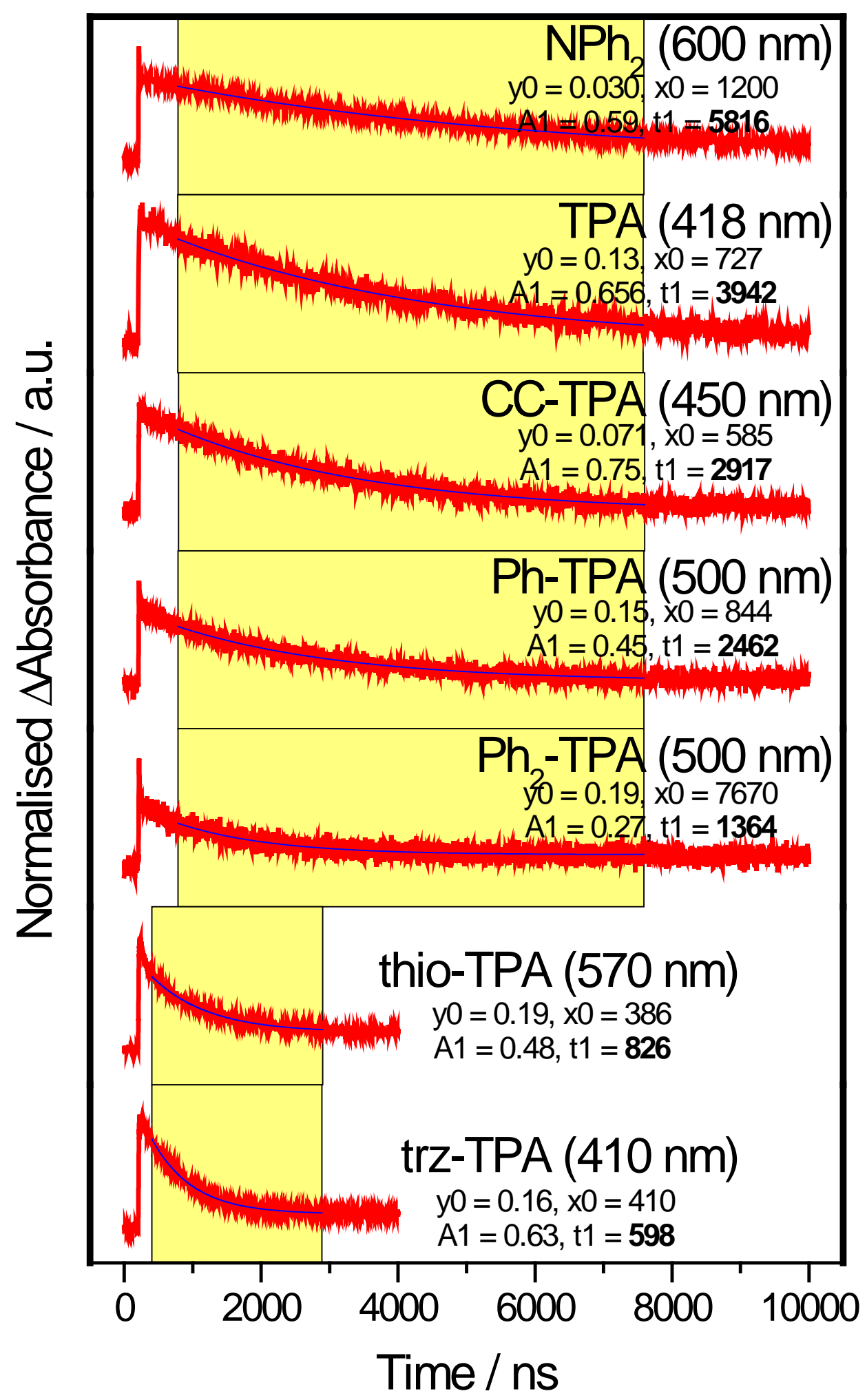

Figure 8 Transient absorption decay profiles of the $\left[\mathrm{ReCl}(\mathrm{CO})_{3}\right]$ complexes at room temperature, recorded in $\mathrm{CH}_{2} \mathrm{Cl}_{2}$ with a

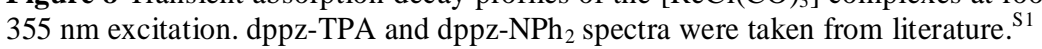


a) dppz-thio-TPA

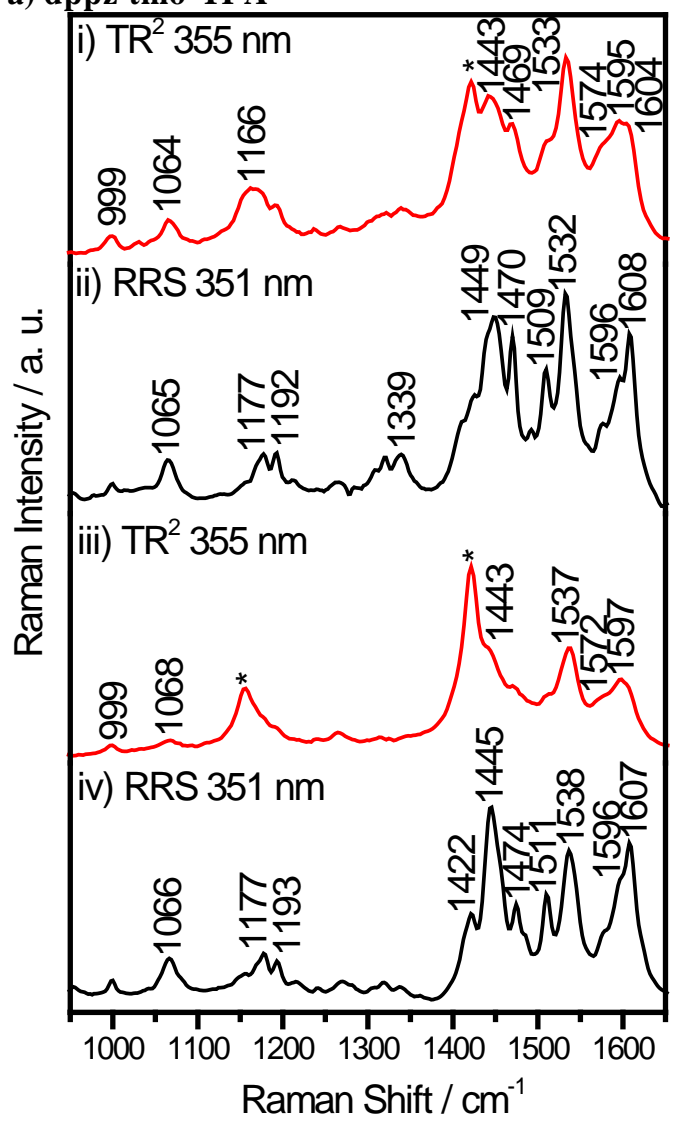

c) dppz-Ph2-TPA

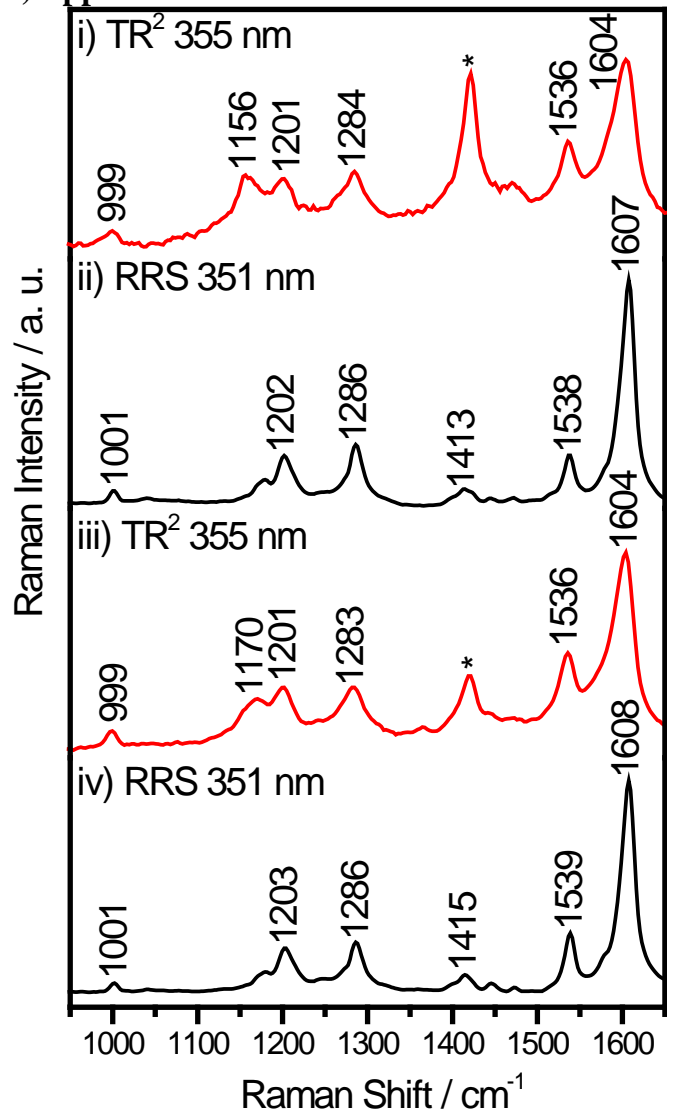

b) dppz-Ph-TPA

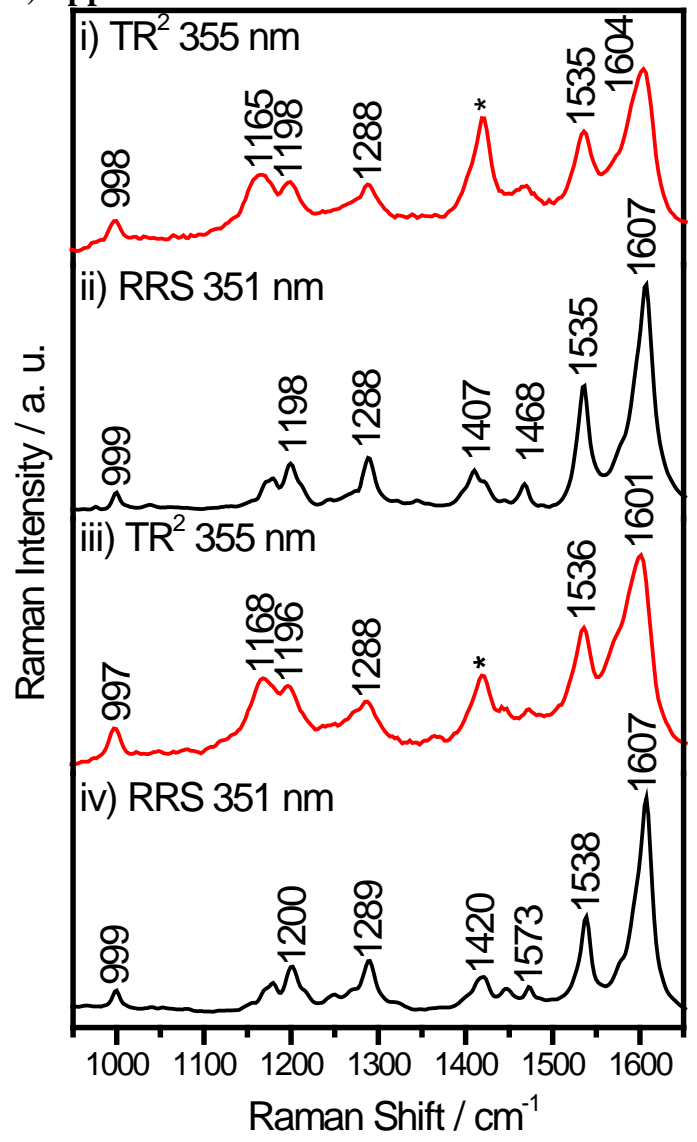

d) dppz-trz-TPA

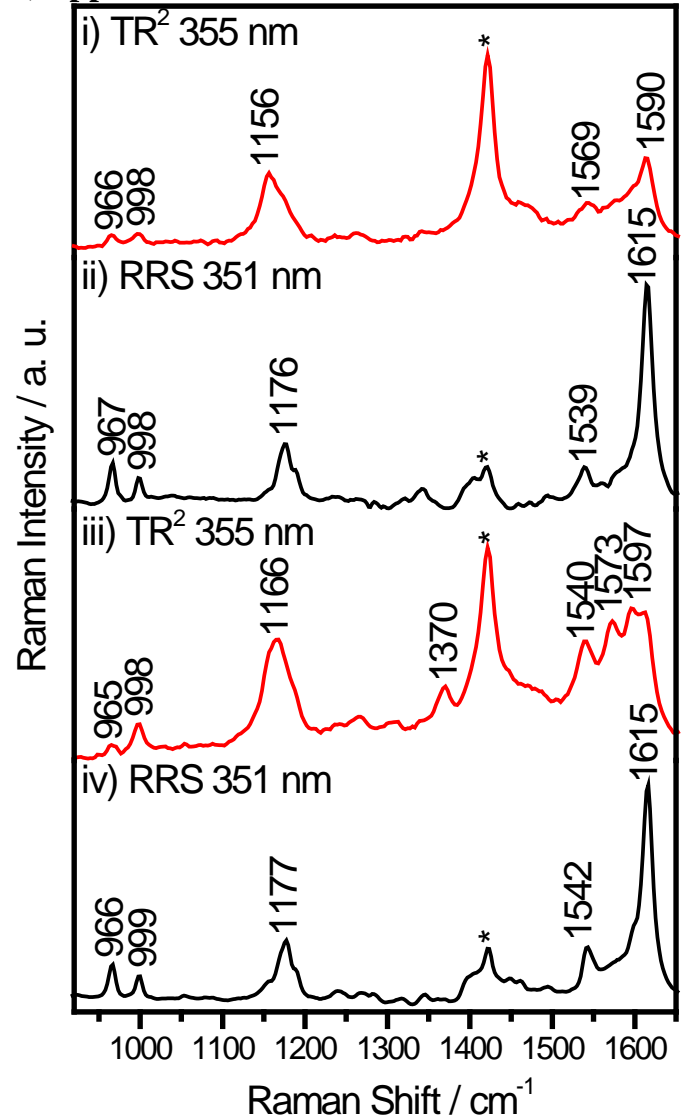

Figure S9 Transient resonance Raman spectra of i) the ligand and iii) the complex compared to the resonance Raman spectra of ii) the ligand and iv) the complex. Spectra were recorded in $\mathrm{CH}_{2} \mathrm{Cl}_{2}$ at $10^{-3} \mathrm{M}$. 
a) $\left[\operatorname{ReCl}(\mathrm{CO})_{3}(\right.$ dppz-thio-TPA $\left.)\right]$

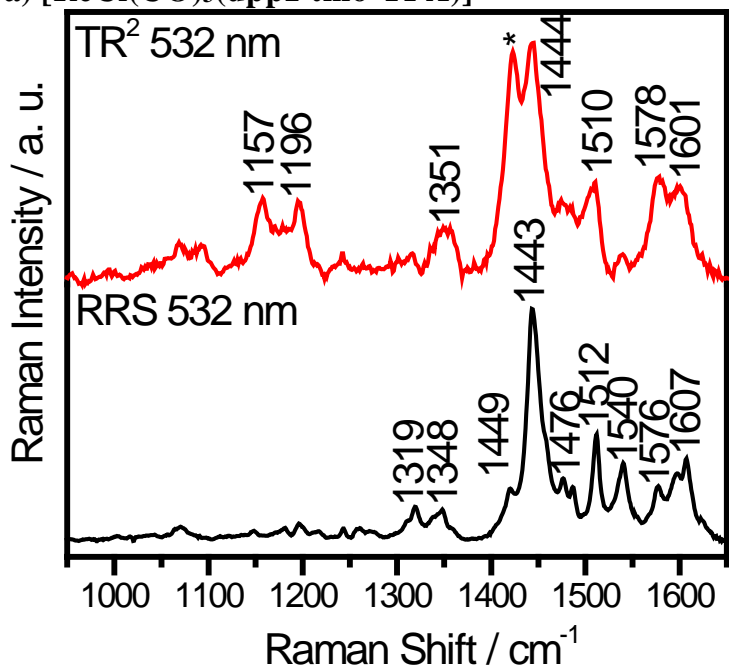

b) $\left[\operatorname{ReCl}(\mathrm{CO})_{3}(\right.$ dppz-Ph-TPA $\left.)\right]$

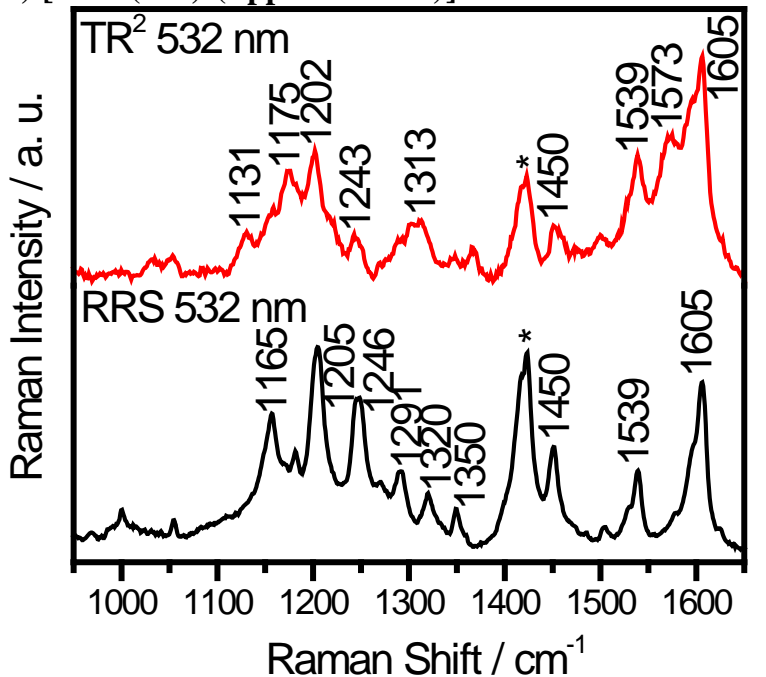

Figure S10 Transient resonance Raman and resonance Raman spectra for a) $\left[\operatorname{ReCl}(\mathbf{C O})_{3}(\mathbf{d p p z}-\right.$ thio-TPA)] and b) $\left[\operatorname{ReCl}(\mathbf{C O})_{3}(\mathbf{d p p z}-\mathbf{P h}-\mathbf{T P A})\right]$. Spectra were recorded with an excitation of $532 \mathrm{~nm}$ in $\mathrm{CH}_{2} \mathrm{Cl}_{2}$ at $10^{-3} \mathrm{M}$.

a) $\left[\operatorname{ReCl}(\mathrm{CO})_{3}\left(\mathrm{dppz}-\mathrm{Ph}_{2}-\mathrm{TPA}\right)\right]$

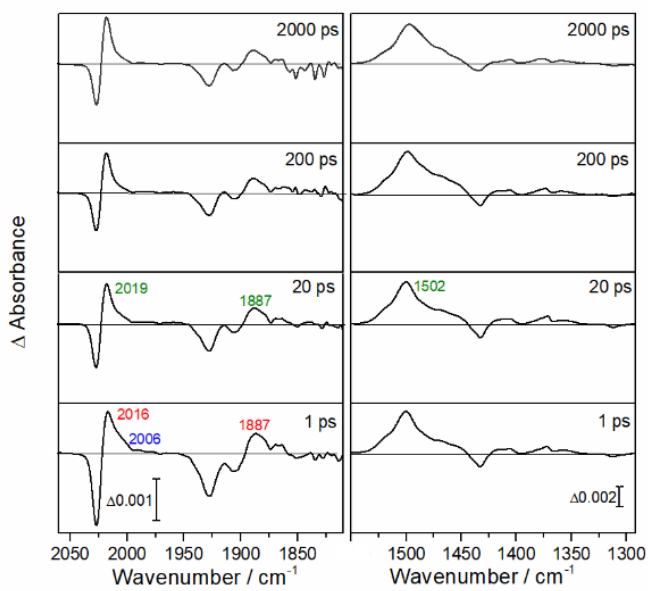

b) $\left[\operatorname{ReCl}(\mathrm{CO})_{3}(\right.$ dppz-trz-TPA $\left.)\right]$

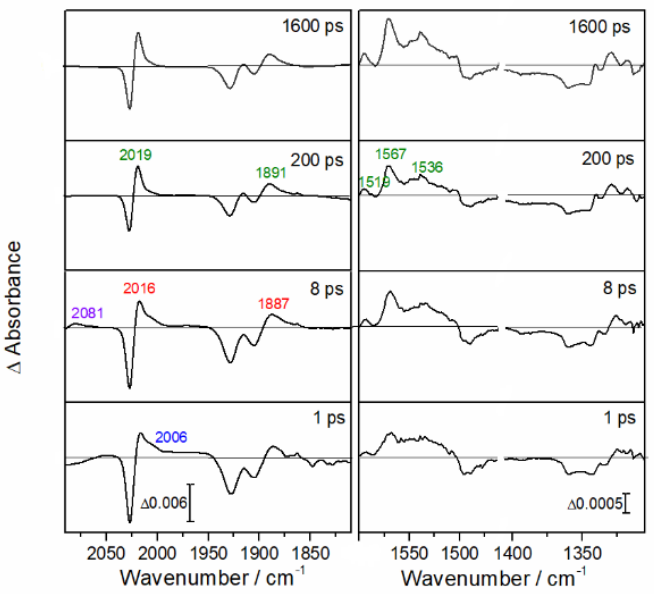

Figure S11 TRIR spectra at several time delays after excitation with a $355 \mathrm{~nm}$ pulse. Spectra were recorded in $\mathrm{CH}_{2} \mathrm{Cl}_{2}$ at $10^{-}$ ${ }^{3} \mathrm{M}$. 


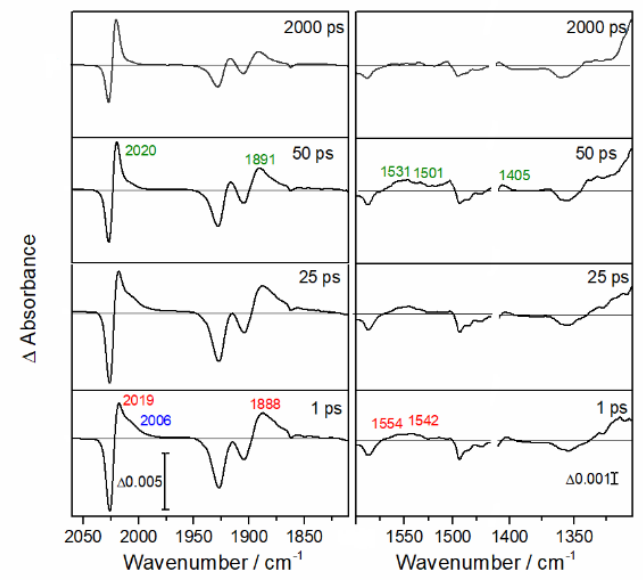

c) $\left[\operatorname{ReCl}(\mathrm{CO})_{3}(\mathrm{dppz}-\right.$ thio-TPA)$]$

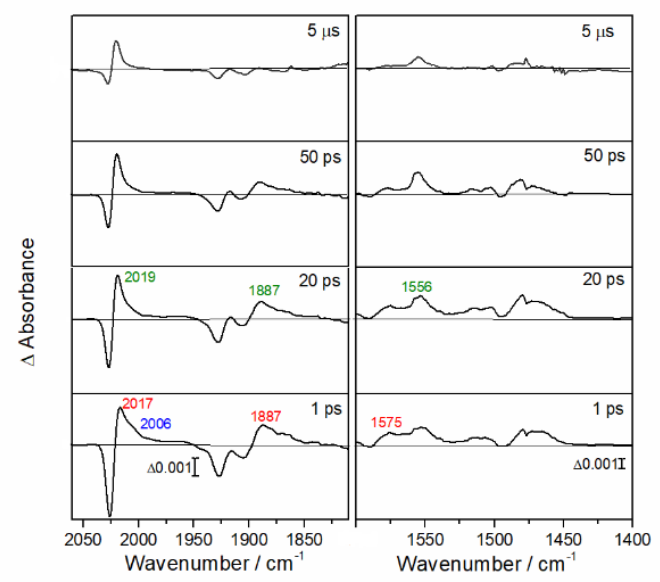

b) $\left[\operatorname{ReCl}(\mathrm{CO})_{3}(\mathrm{dppz}-\mathrm{TPA})\right]$

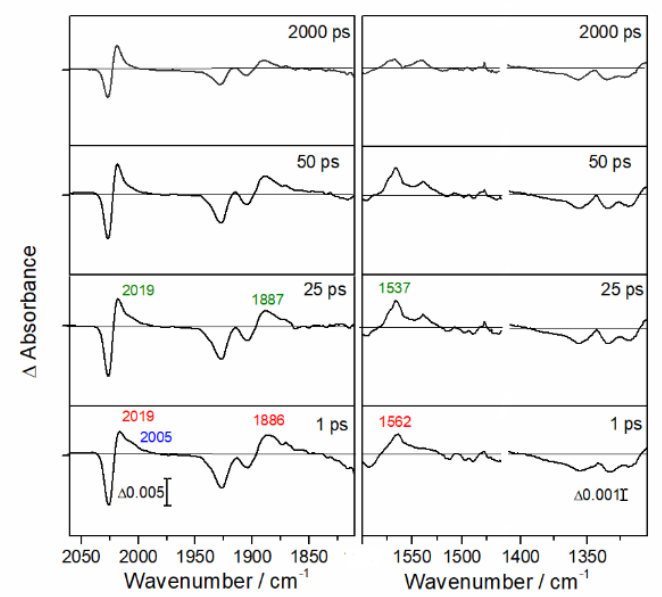

d) $\left[\mathrm{ReCl}(\mathrm{CO})_{3}(\mathrm{dppz}-\mathrm{Ph}-\mathrm{TPA})\right]$

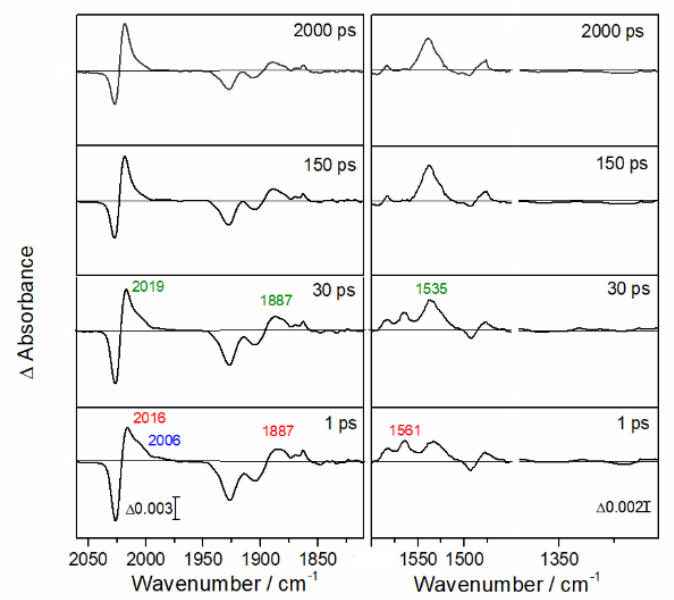

Figure S12 TRIR spectra at several time delays after excitation with a $355 \mathrm{~nm}$ pulse. Spectra were recorded in $\mathrm{CH}_{2} \mathrm{Cl}_{2}$ at $10^{-}$ ${ }^{3} \mathrm{M}$. 


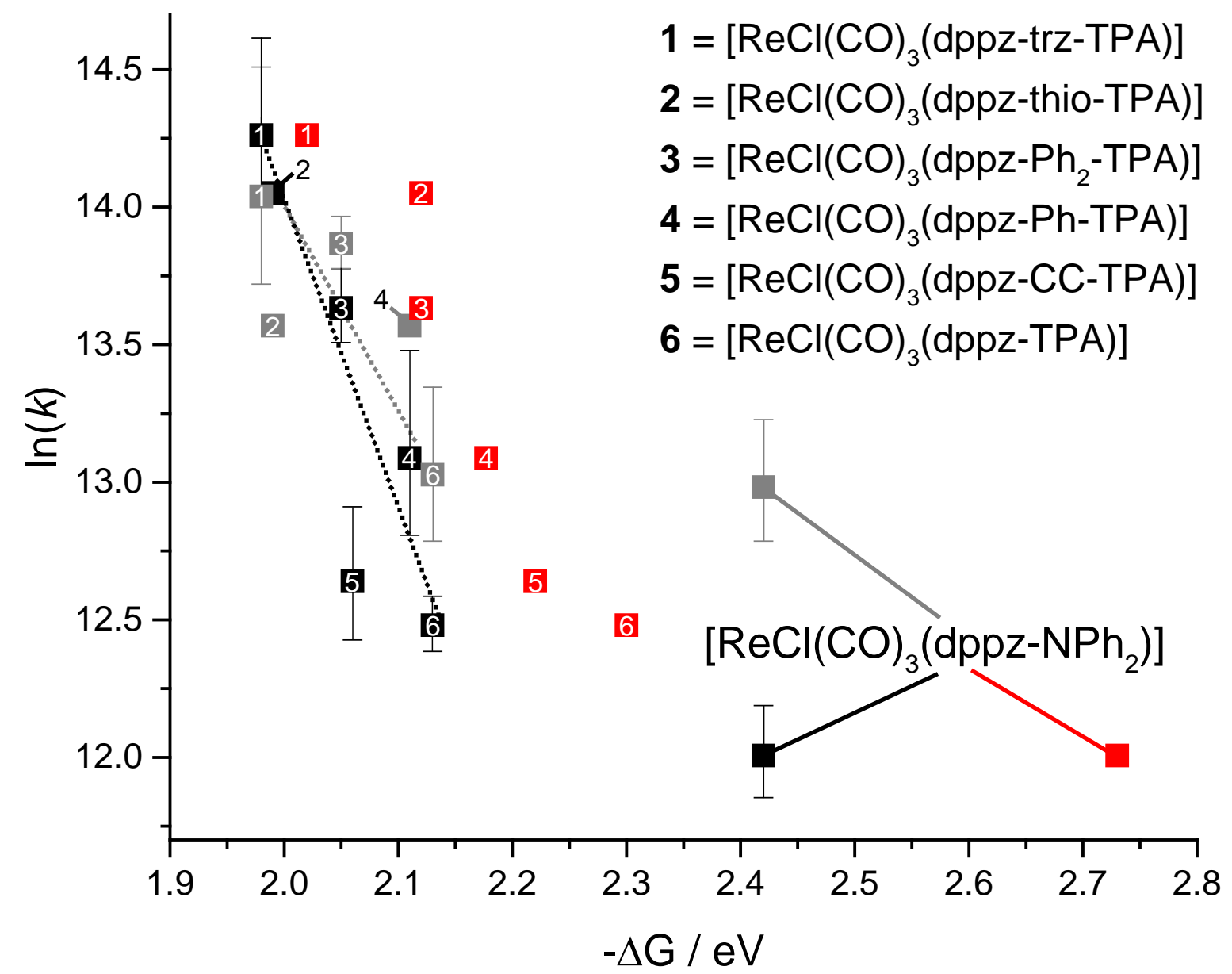

Figure S13 Tentative relationship between $-\Delta \mathrm{G}$ and $\ln (k)$ for the complexes. For experimental TA (black) and TR-IR (grey) $-\Delta G$ values are calculated from electrochemical data. For B3LYP calculated $-\Delta G$ values (red) the $\ln (\mathrm{k})$ values are TA values 


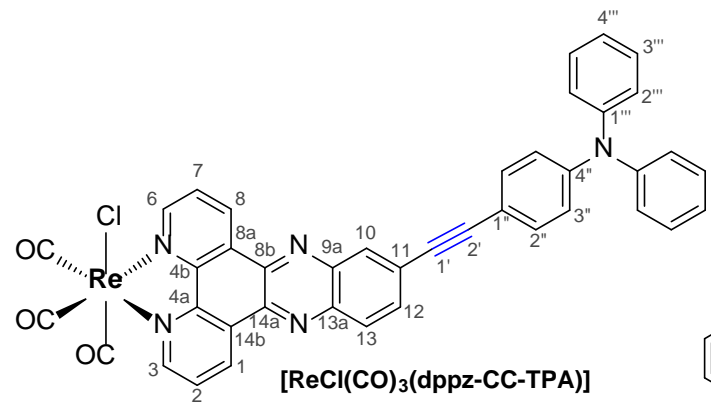

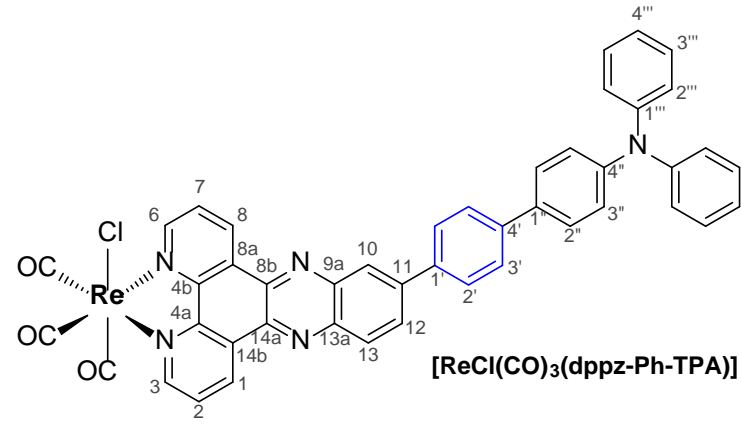

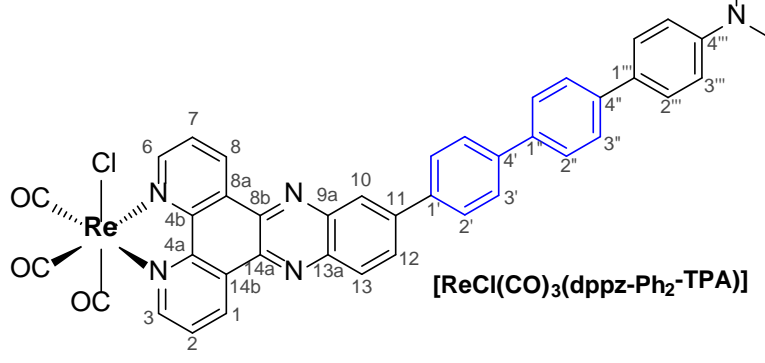

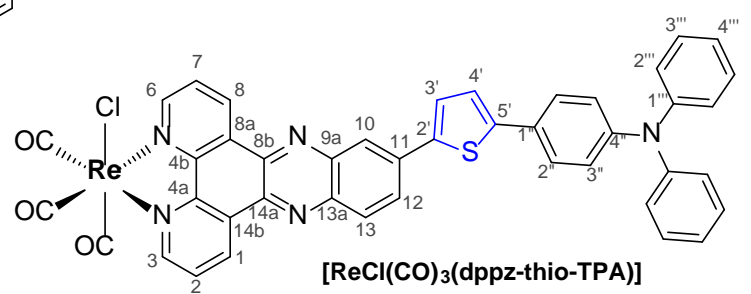

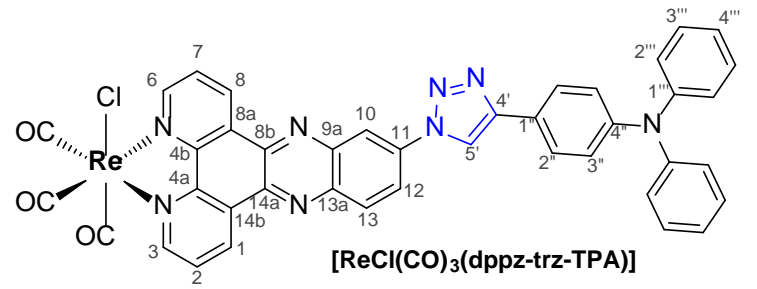

Figure S5. Atom numbering schemes for the new $\left[\mathrm{ReCl}(\mathrm{CO})_{3}(\mathrm{dppz}-\right.$ linker-TPA)] complexes reported.

\section{References}

S1 Li, Y.; Cao, Y.; Gao, J.; Wang, D.; Yu, G.; Heeger, A. J., Electrochemical properties of luminescent polymers and polymer light-emitting electrochemical cells. Synth. Met. 1999, 99, 243-248.

S2 Adams, B. S.; Shillito, G. E.; van der Salm, H.; Horvath, R.; Larsen, C. B.; Sun, X.-Z.; Lucas, N. T.; George, M. W.; Gordon, K. C., Alteration of Intraligand Donor-Acceptor Interactions Through Torsional Connectivity in Substituted Re-dppz Complexes. Inorg. Chem. 2017, 56, 12967-12977. 